\title{
Review Article \\ Evidence and Search for Sterile Neutrinos at Accelerators
}

\author{
W. C. Louis \\ Los Alamos National Laboratory, Los Alamos, NM 87545, USA \\ Correspondence should be addressed to W. C. Louis; louis@lanl.gov
}

Received 11 June 2013; Accepted 13 August 2013

Academic Editor: Leslie Camilleri

Copyright (C) 2013 W. C. Louis. This is an open access article distributed under the Creative Commons Attribution License, which permits unrestricted use, distribution, and reproduction in any medium, provided the original work is properly cited.

The LSND short-baseline neutrino experiment has published evidence for antineutrino oscillations at a mass scale of $\sim 1 \mathrm{eV}^{2}$. The MiniBooNE experiment, designed to test this evidence for oscillations at an order of magnitude higher neutrino energy and distance, observes excesses of events in both neutrino mode and antineutrino mode. While the MiniBooNE neutrino excess has a neutrino energy spectrum that is softer than expected from LSND, the MiniBooNE antineutrino excess is consistent with neutrino oscillations and with the LSND oscillation signal. When combined with oscillation measurements at the solar and atmospheric mass scales, assuming that the LSND and MiniBooNE signals are due to neutrino oscillations, these experiments imply the existence of more than three neutrino mass states and, therefore, one or more sterile neutrinos. Such sterile neutrinos, if proven to exist, would have a big impact on particle physics, nuclear physics, and astrophysics and would contribute to the dark matter of the universe. Future experiments under construction or proposed at Fermilab, ORNL, CERN, and in Japan will provide a definitive test of shortbaseline neutrino oscillations and will have the capability of proving the existence of sterile neutrinos.

\section{Introduction}

Neutrino oscillations have been clearly observed at the solar mass scale of $\Delta m^{2} \sim 7.5 \times 10^{-5} \mathrm{eV}^{2}$ and the atmospheric mass scale of $\Delta m^{2} \sim 2.4 \times 10^{-3} \mathrm{eV}^{2}[1]$. However, evidence for neutrino oscillations at the $\Delta m^{2} \sim 1 \mathrm{eV}^{2}$ mass scale has also been given by the LSND [2-6] and MiniBooNE [79] experiments. If the signals reported by LSND and MiniBooNE are indeed due to neutrino oscillations, then there is a problem with the three-neutrino paradigm, because it is not possible to explain oscillations at the three different mass scales with only three types of neutrinos. In order to solve this problem, additional "sterile" neutrinos have been proposed [10-14]. Other explanations include, for example, Lorentz violation $[15,16]$ and sterile neutrino decay $[17,18]$. These additional neutrinos would need to be sterile to the weak interaction due to measurements of the width of the $Z^{\circ}$ boson, which determine that there are three and only three active neutrinos [19]. The evidence for neutrino oscillations from LSND and MiniBooNE will be discussed in the following sections, followed by a discussion of global fits to the world data and future experiments, which will have the capability of proving whether short-baseline neutrino oscillations at the $\sim 1 \mathrm{eV}^{2}$ mass scale and light, sterile neutrinos exist in nature.

\section{The LSND Experiment}

The LSND experiment [20] was designed to search for $\bar{\nu}_{\mu} \rightarrow$ $\bar{\nu}_{e}$ oscillations with high sensitivity and to measure $\nu C$ crosssections. A photograph of the inside of the detector tank is shown in Figure 1. The main characteristics of the LSND experiment are given in Table 1. LSND had the advantage of a very high proton intensity, a large detector mass, and good particle identification. LSND made use of a high-intensity, $798 \mathrm{MeV}$ proton beam that interacted in an absorber to produce a large number of pions.

2.1. Neutrino Beam. LSND made use of the LAMPF accelerator, which was an intense source of low energy neutrinos produced with a proton current of $1 \mathrm{~mA}$ at $798 \mathrm{MeV}$ kinetic energy. For the 1993-1995 running period, the production target consisted of a $30 \mathrm{~cm}$ long water target $(20 \mathrm{~cm}$ in 1993) followed by a water-cooled $\mathrm{Cu}$ beam dump, while for the 1996-1998 running period, the production target was 
TABLE 1: The main characteristics of the LSND and MiniBooNE experiments. Also the estimated number of neutrino background events and signal events for $100 \% v_{\mu} \rightarrow v_{e}$ transmutation are shown. The $R_{\gamma}>10$ requirement is assumed for the LSND events.

\begin{tabular}{lccc}
\hline Property & LSND & MiniBooNE $\nu$ & MiniBooNE $\bar{\nu}$ \\
\hline Proton energy & $798 \mathrm{MeV}$ & $8000 \mathrm{MeV}$ & $8000 \mathrm{MeV}$ \\
Proton intensity & $1000 \mu \mathrm{A}$ & $4 \mu \mathrm{A}$ & $4 \mu \mathrm{A}$ \\
Proton beam power & $798 \mathrm{~kW}$ & $32 \mathrm{~kW}$ & $32 \mathrm{~kW}$ \\
Protons on target & $28,896 \mathrm{C}$ & $104 \mathrm{C}$ & $181 \mathrm{C}$ \\
Duty factor & $6 \times 10^{-2}$ & $8 \times 10^{-6}$ & $8 \times 10^{-6}$ \\
Total mass & $167 \mathrm{t}$ & $806 \mathrm{t}$ & $806 \mathrm{t}$ \\
Neutrino distance & $30 \mathrm{~m}$ & $541 \mathrm{~m}$ & $541 \mathrm{~m}$ \\
$100 \% \nu_{\mu} \rightarrow \nu_{e}$ events & 12,987 & 89,615 & 38,462 \\
$\nu$ background events & 16.9 & 797.7 & 398.2 \\
\hline
\end{tabular}

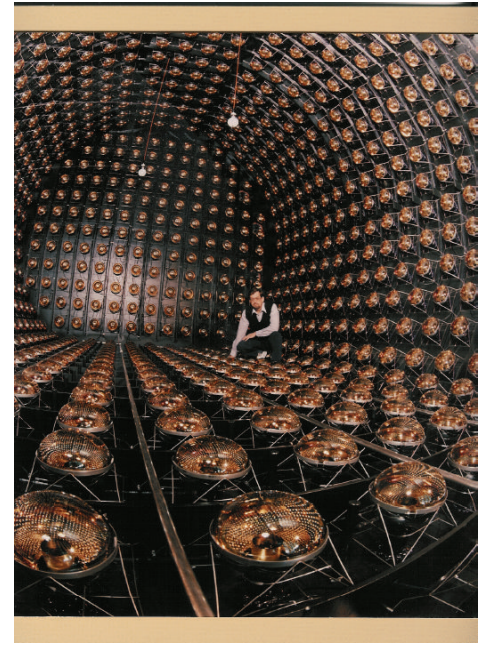

FIgURE 1: A photograph of the inside of the LSND detector tank.

reconfigured with the water target replaced by a close-packed, high- $Z$ target. The resulting decay-at-rest (DAR) neutrino fluxes are well understood because almost all detectable neutrinos arise from $\pi^{+}$or $\mu^{+}$decay; $\pi^{-}$and $\mu^{-}$that stop are readily captured in the Fe of the shielding and $\mathrm{Cu}$ of the beam stop $[21,22]$. The production of kaons or heavier mesons is negligible at these proton energies. The $\bar{\nu}_{e}$ flux is calculated to be only $\sim 8 \times 10^{-4}$ as large as the $\bar{v}_{\mu}$ flux in the $20<E_{\gamma}<52.8 \mathrm{MeV}$ energy range, so that the observation of a $\bar{\nu}_{e}$ event rate significantly above the calculated background would be evidence for $\bar{\nu}_{\mu} \rightarrow \bar{\nu}_{e}$ oscillations. Figure 2 shows the neutrino energy spectra from $\pi^{+}$and $\mu^{+}$DAR.

2.2. Detector. The LSND detector [20] consisted of an approximately cylindrical tank $8.3 \mathrm{~m}$ long by $5.7 \mathrm{~m}$ in diameter. A schematic drawing of the detector is shown in Figure 3. The center of the detector was $30 \mathrm{~m}$ away from the neutrino source. On the inside surface of the tank, 1220 8-inch Hamamatsu PMTs covered 25\% of the area with photocathode. The tank was filled with $167 \mathrm{t}$ of liquid scintillator consisting of mineral oil and $0.031 \mathrm{~g} / \mathrm{L}$ of b-PBD. This low scintillator concentration allows the detection of both Cherenkov light and scintillation light and yields an attenuation length of more than $20 \mathrm{~m}$ for wavelengths greater than $400 \mathrm{~nm}$ [23]. A typical $45 \mathrm{MeV}$ electron created in the detector produced a total of $\sim 1500$ photoelectrons, of which $\sim 280$ photoelectrons were in the Cherenkov cone. PMT time and pulse-height signals were used to reconstruct particle tracks with an average RMS position resolution of $\sim 14 \mathrm{~cm}$, an angular resolution of $\sim 12^{\circ}$, and an energy resolution of $\sim 7 \%$ at the Michel endpoint of $52.8 \mathrm{MeV}$. The Cherenkov cone for relativistic particles and the time distribution of the light, which is broader for nonrelativistic particles [20], gave excellent separation between electrons and particles below Cherenkov threshold. Identification of neutrons was accomplished through the detection of the $2.2 \mathrm{MeV} \gamma$ from neutron capture on a free proton. The veto shield enclosed the detector on all sides except the bottom. Additional counters were placed below the veto shield after the 1993 run to reduce cosmic-ray background entering through the bottom support structure. The main veto shield [24] consisted of a $15 \mathrm{~cm}$ layer of liquid scintillator in an external tank and $15 \mathrm{~cm}$ of lead shot in an internal tank. This combination of active and passive shielding tagged cosmic-ray muons that stopped in the lead shot. A veto inefficiency of $<10^{-5}$ was achieved for incident charged particles.

2.3. Oscillation Results. The primary oscillation search in LSND is for $\bar{\nu}_{\mu} \rightarrow \bar{\nu}_{e}$ oscillations, where the $\bar{\nu}_{\mu}$ arise from $\mu^{+}$DAR in the beam stop and the $\bar{\nu}_{e}$ are identified through the reaction $\bar{\nu}_{e} p \rightarrow e^{+} n$. This reaction allows a two-fold signature of a positron with a $52.8 \mathrm{MeV}$ endpoint and a correlated $2.2 \mathrm{MeV} \gamma$ from neutron capture on a free proton. There are only two significant neutrino backgrounds with a positron/electron and a correlated neutron. The first background is from $\mu^{-}$DAR in the beam stop followed by $\bar{\nu}_{e} p \rightarrow e^{+} n$ scattering in the detector. This background is highly suppressed due to the requirements that a $\pi^{-}$be produced, the $\pi^{-}$decays in flight, and the $\mu^{-}$decays at rest prior to capture. The second background is from $\pi^{-}$ decay in flight (DIF) in the beam stop followed by $\bar{\nu}_{\mu} p \rightarrow$ $\mu^{+} n$ scattering in the detector. Additional contributions are from $\bar{\nu}_{\mu} C \rightarrow \mu^{+} n X$ and $\nu_{\mu} C \rightarrow \mu^{-} n X$ scattering. This background will mimic the oscillation reaction if the $\mu^{+}$is 


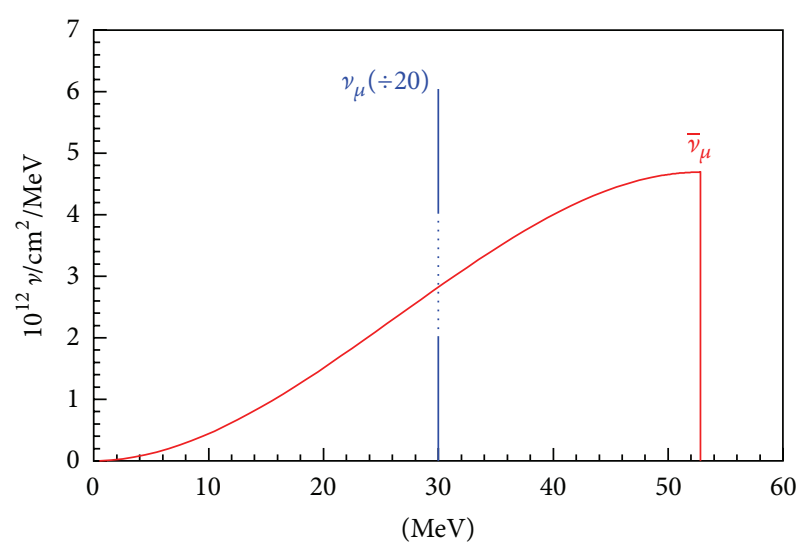

(a)

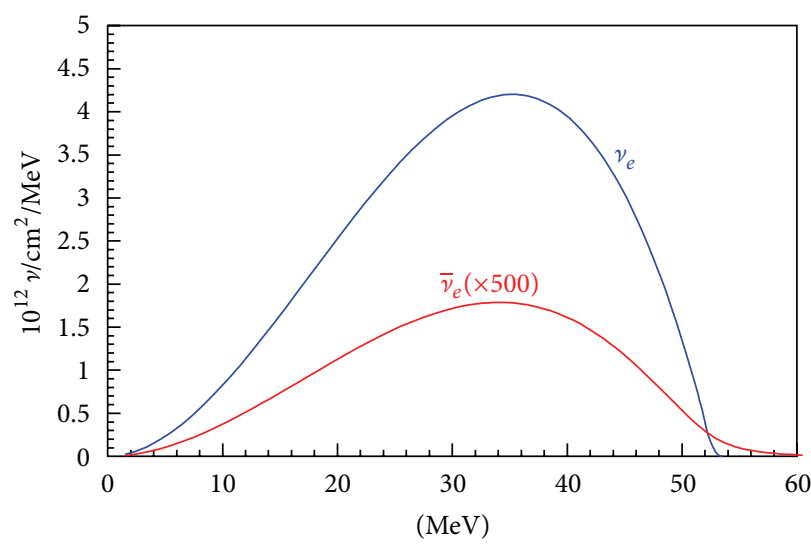

(b)

FIgURE 2: The neutrino energy spectra from $\pi^{+}$and $\mu^{+}$DAR.
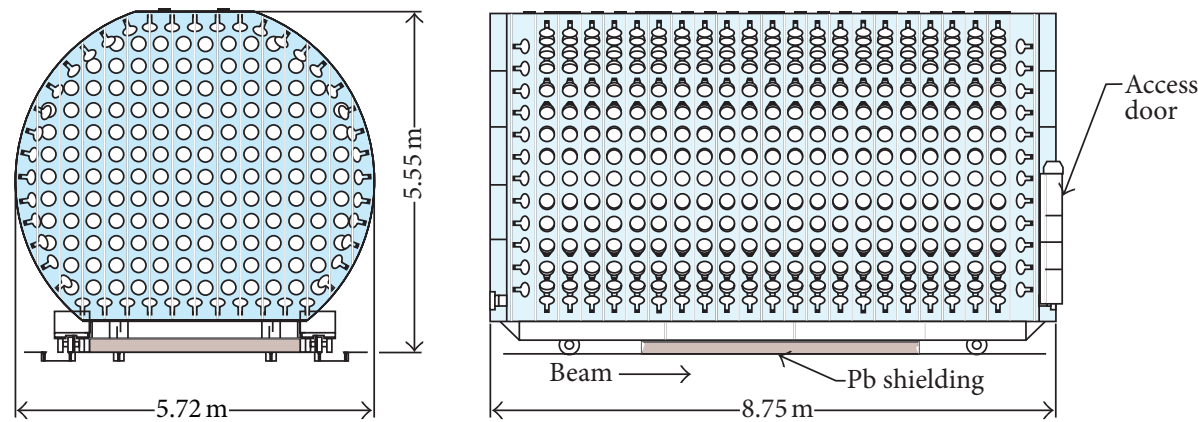

FIGURE 3: A schematic drawing of the LSND detector.

sufficiently low in energy that it is below the threshold of 18 hit PMTs, corresponding to $E_{\mu}<4 \mathrm{MeV}$. Table 2 shows the estimated number of events in the $20<E_{e}<60 \mathrm{MeV}$ energy range satisfying the electron selection criteria for $100 \% \bar{\nu}_{\mu} \rightarrow$ $\bar{\nu}_{e}$ transmutation and for the two beam-related backgrounds with neutrons.

Table 3 shows the LSND statistics for events that satisfy the selection criteria for the primary $\bar{\nu}_{\mu} \rightarrow \bar{\nu}_{e}$ oscillation search. An excess of events is observed over that expected from beam-off and neutrino background that is consistent with neutrino oscillations [2-6]. A $\chi^{2}$ fit to the $R_{\gamma}$ distribution, as shown in Figure 4 , gives $f_{c}=0.0567 \pm 0.0108$ $\left(\chi^{2}=10.7 / 9 \mathrm{DOF}\right)$, which leads to a beam on-off excess of $117.9 \pm 22.4$ events with a correlated neutron. $R_{\gamma}$ is the likelihood that the $\gamma$ is a correlated $\gamma$ from neutron capture divided by the likelihood that the $\gamma$ is an accidental $\gamma$, while $f_{c}$ is the fraction of electron candidate events with a correlated $\gamma$. Subtracting the neutrino background from $\mu^{-}$DAR followed by $\bar{\nu}_{e} p \rightarrow e^{+} n$ scattering (19.5 \pm 3.9 events) and $\pi^{-}$DIF followed by $\bar{\nu}_{\mu} p \rightarrow \mu^{+} n$ scattering (10.5 \pm 4.6 events) (this background also includes contributions from $\bar{\nu}_{\mu} \mathrm{C} \rightarrow$ $\mu^{+} n X$ and $\left.\nu_{\mu} C \rightarrow \mu^{-} n X\right)$ leads to a total excess of $87.9 \pm$ $22.4 \pm 6.0$ events. This excess corresponds to an oscillation probability of $(0.264 \pm 0.067 \pm 0.045) \%$, where the first error is statistical and the second error is the systematic error
TABLE 2: The LSND estimated number of events in the $20<E_{e}<$ $60 \mathrm{MeV}$ energy range due to $100 \% \bar{\nu}_{\mu} \rightarrow \bar{\nu}_{e}$ transmutation and the two beam-related backgrounds with neutrons, $\mu^{-}$DAR in the beam stop followed by $\bar{v}_{e} p \rightarrow e^{+} n$ scattering in the detector and $\pi^{-}$DIF in the beam stop followed by $\bar{\nu}_{\mu} p \rightarrow \mu^{+} n$ scattering. The events must satisfy the electron selection criteria, but no correlated $\gamma$ requirement is imposed.

\begin{tabular}{lcc}
\hline Neutrino source & Reaction & Number of events \\
\hline$\mu^{+}$DAR & $100 \% \bar{\nu}_{\mu} \rightarrow \bar{\nu}_{e}$ & $33300 \pm 3300$ \\
$\mu^{-}$DAR & $\bar{\nu}_{e} p \rightarrow e^{+} n$ & $19.5 \pm 3.9$ \\
$\pi^{-}$DIF & $\bar{\nu}_{\mu} p \rightarrow \mu^{+} n$ & $10.5 \pm 4.6$ \\
\hline
\end{tabular}

arising from uncertainties in the backgrounds, neutrino flux (7\%), $e^{+}$efficiency (7\%), and $\gamma$ efficiency (7\%).

A clean sample of oscillation candidate events can be obtained by requiring $R_{\gamma}>10$, where, as shown in Table 3, the beam on-off excess is $49.1 \pm 9.4$ events, while the estimated neutrino background is only $16.9 \pm 2.3$ events. Figure 5 displays the energy distribution of events with $R_{\gamma}>$ 10. The shaded regions show the combination of neutrino background plus neutrino oscillations at low $\Delta m^{2}$. The data agree well with the oscillation hypothesis. Finally, Figure 6 shows the $L / E_{\nu}$ distribution for events with $R_{\gamma}>10$ and 
TABLE 3: Numbers of LSND beam-on events that satisfy the selection criteria for the primary $\bar{\nu}_{\mu} \rightarrow \bar{\nu}_{e}$ oscillation search with $R_{\gamma}>1, R_{\gamma}>10$, and $R_{\gamma}>100$. Also the beam-off background, the estimated neutrino background, and the excess of events that is consistent with neutrino oscillations are shown.

\begin{tabular}{lcccc}
\hline Selection & $\begin{array}{c}\text { Beam-on } \\
\text { events }\end{array}$ & $\begin{array}{c}\text { Beam-off } \\
\text { background }\end{array}$ & $\nu$ background & Event excess \\
\hline$R_{\gamma}>1$ & 205 & $106.8 \pm 2.5$ & $39.2 \pm 3.1$ & $59.0 \pm 14.5 \pm 3.1$ \\
$R_{\gamma}>10$ & 86 & $36.9 \pm 1.5$ & $16.9 \pm 2.3$ & $32.2 \pm 9.4 \pm 2.3$ \\
$R_{\gamma}>100$ & 27 & $8.3 \pm 0.7$ & $5.4 \pm 1.0$ & $13.3 \pm 5.2 \pm 1.0$ \\
\hline
\end{tabular}

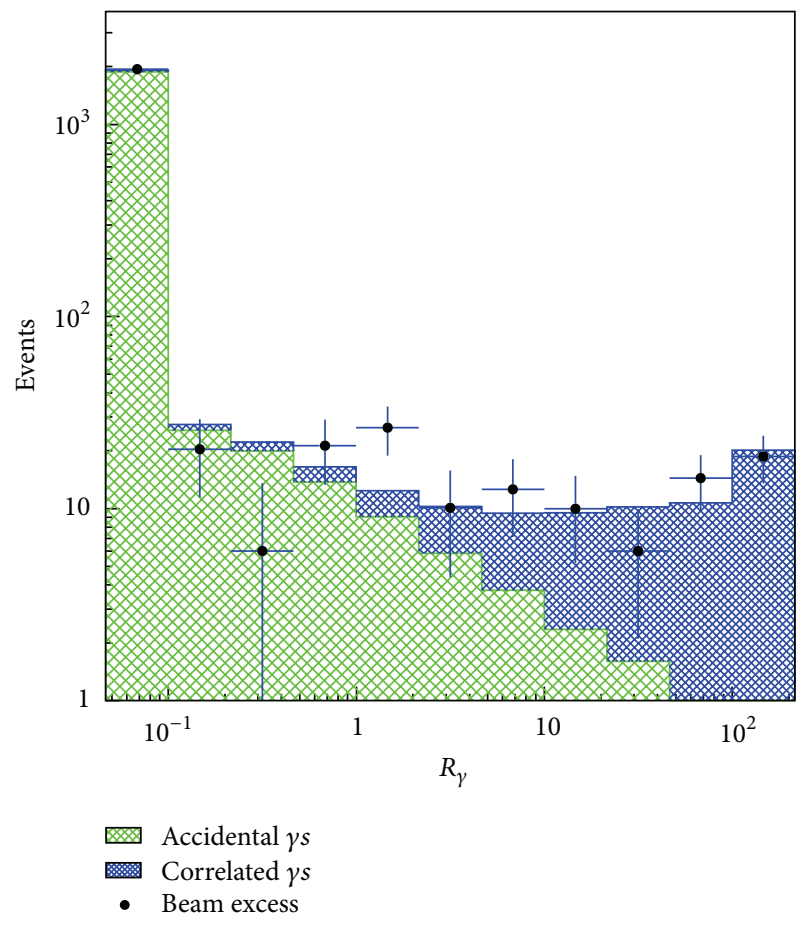

FIGURE 4: The LSND $R_{\gamma}$ distribution for events that satisfy the selection criteria for the primary $\bar{\nu}_{\mu} \rightarrow \bar{\nu}_{e}$ oscillation search.

$20<E_{e}<60 \mathrm{MeV}$, where $L$ is the distance travelled by the neutrino in meters and $E_{v}$ is the neutrino energy in $\mathrm{MeV}$ determined from the measured positron energy and angle with respect to the neutrino beam. The data agree well with the expectation from neutrino background plus neutrino oscillations at low $\Delta m^{2}\left(\chi^{2}=4.9 / 8\right.$ D.O.F. $)$ or high $\Delta m^{2}$ $\left(\chi^{2}=5.8 / 8\right.$ D.O.F. $)$.

The $\left(\sin ^{2} 2 \theta, \Delta m^{2}\right)$ likelihood $(\mathscr{L})$ fitter is applied to beam-on events in the final oscillation sample and calculates a likelihood in the $\left(\sin ^{2} 2 \theta, \Delta m^{2}\right)$ plane in order to extract the favored oscillation parameters. The $\mathscr{L}$ product in the $\left(\sin ^{2} 2 \theta, \Delta m^{2}\right)$ plane is formed over the individual beam-on events that pass the oscillation cuts. This threedimensional contour is sliced to arrive finally at the LSND allowed oscillation region. The beam-related backgrounds are determined from Monte Carlo (MC) event samples for each individual background contribution. The MC contains the trigger simulation and generally very well reproduces the tank response to all particles of interest. Agreement between the data and MC is excellent.

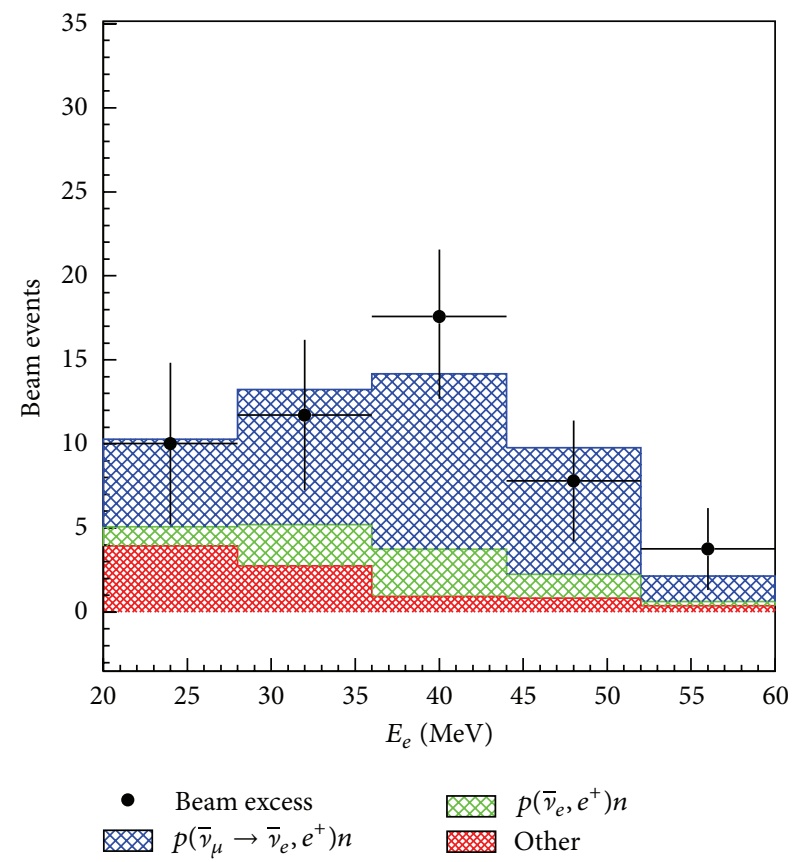

FIGURE 5: The LSND electron energy distribution for events with $R_{\gamma}>10$. The shaded region shows the expected distribution from a combination of neutrino background plus neutrino oscillations at low $\Delta m^{2}$.

The $\left(\sin ^{2} 2 \theta, \Delta m^{2}\right)$ oscillation parameter fit for the entire data sample, $20<E_{e}<200 \mathrm{MeV}$, is shown in Figure 7 . The fit includes both $\bar{\nu}_{\mu} \rightarrow \bar{\nu}_{e}$ and $\nu_{\mu} \rightarrow \nu_{e}$ oscillations (although the latter contribution is very small), as well as all known neutrino backgrounds. The inner and outer regions correspond to $90 \%$ and $99 \%$ CL allowed regions, while the curves are $90 \%$ CL limits from the Bugey reactor experiment [25] and the KARMEN experiment at ISIS [26]. Note that the Bugey limit has been relaxed recently due to the reactor neutrino anomaly [27]. The most favored allowed region is the band from 0.2 to $2.0 \mathrm{eV}^{2}$, although a region around $7 \mathrm{eV}^{2}$ is also possible.

\section{The MiniBooNE Experiment}

The MiniBooNE experiment was designed to test the LSND neutrino oscillation signal with a neutrino energy and distance that were an order of magnitude higher than LSND but with an $L / E$ ratio that was approximately the same as for 


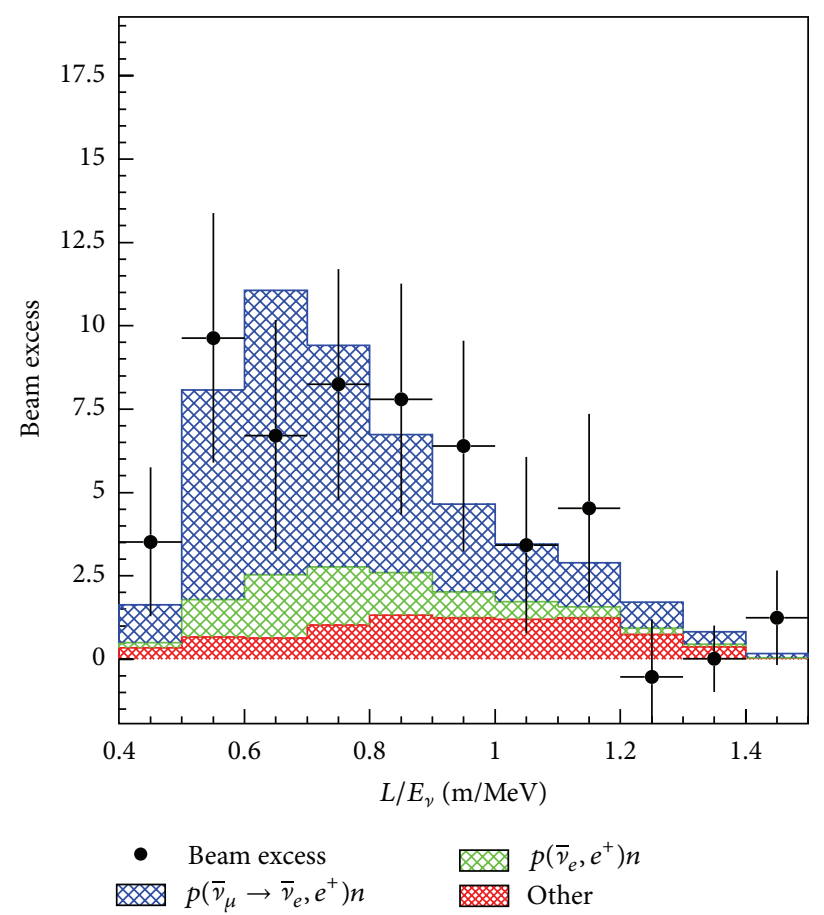

Figure 6: The LSND $L / E_{\nu}$ distribution for events with $R_{\gamma}>10$ and $20<E_{e}<60 \mathrm{MeV}$, where $L$ is the distance travelled by the neutrino in meters and $E_{\gamma}$ is the neutrino energy in $\mathrm{MeV}$. The data agree well with the expectation from neutrino background and neutrino oscillations at low $\Delta m^{2}$.

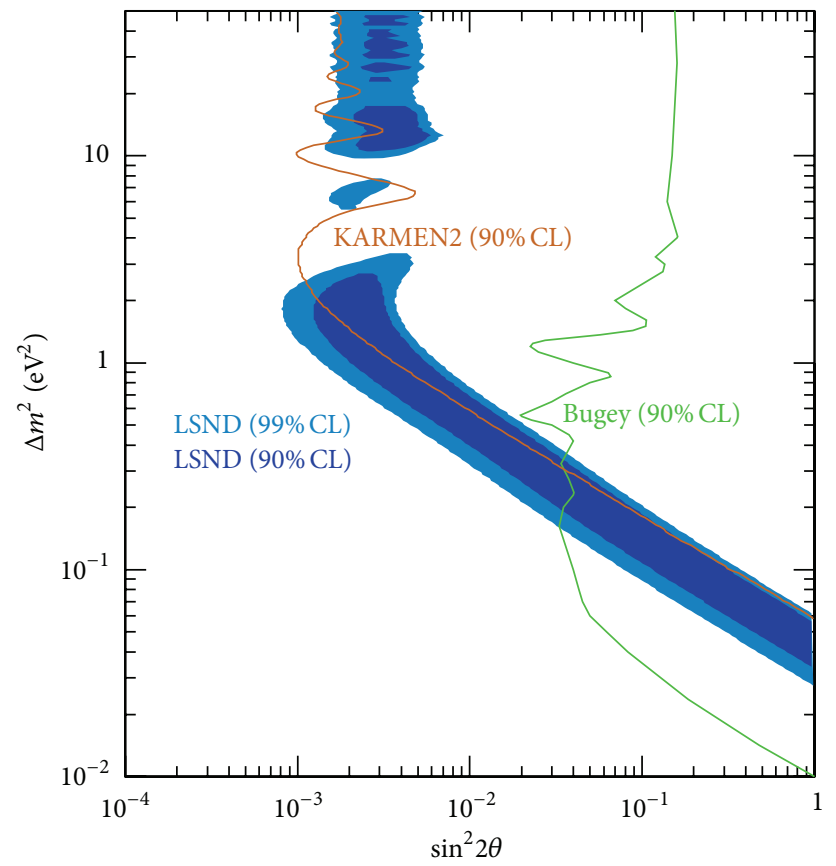

FIGURE 7: The $\left(\sin ^{2} 2 \theta, \Delta m^{2}\right)$ oscillation parameter fit for the entire LSND data sample, $20<E_{e}<200 \mathrm{MeV}$. The inner and outer regions correspond to $90 \%$ and $99 \%$ CL allowed regions, while the curves are 90\% CL limits from the Bugey reactor experiment and the KARMEN experiment at ISIS. 


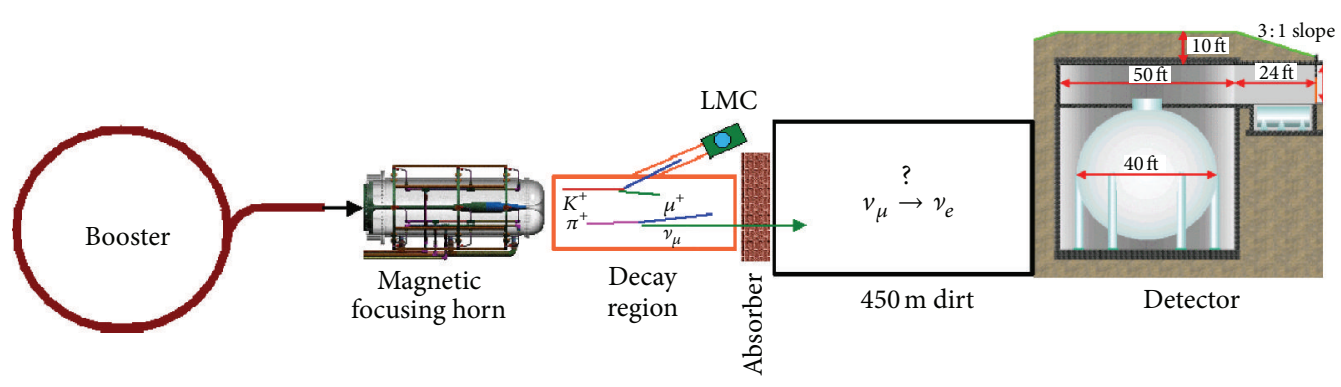

FIgURE 8: A schematic drawing of the MiniBooNE experiment.

LSND. Due to the higher neutrino energies, the backgrounds and systematic errors are completely different between the two experiments.

3.1. Neutrino Beam. A schematic drawing of the MiniBooNE experiment at FNAL is shown in Figure 8. The experiment is fed by $8 \mathrm{GeV}$ kinetic energy protons from the Booster that interact in a $71 \mathrm{~cm}$ long Be target located at the upstream end of a magnetic focusing horn. The horn pulses with a current of $174 \mathrm{kA}$ and, depending on the polarity, either focuses $\pi^{+}$and $K^{+}$and defocuses $\pi^{-}$and $K^{-}$to form a pure neutrino beam or focuses $\pi^{-}$and $K^{-}$and defocuses $\pi^{+}$ and $K^{+}$to form a somewhat pure antineutrino beam. The produced pions and kaons decay in a $50 \mathrm{~m}$ long pipe, and a fraction of the neutrinos and antineutrinos [28] interact in the MiniBooNE detector, which is located $541 \mathrm{~m}$ downstream of the Be target. For the MiniBooNE results presented here, a total of $6.46 \times 10^{20}$ POT were collected in neutrino mode and $11.27 \times 10^{20}$ POT were collected in antineutrino mode.

3.2. Detector. The MiniBooNE detector [29] consists of a $12.2 \mathrm{~m}$ diameter spherical tank filled with approximately 806 tons of mineral oil $\left(\mathrm{CH}_{2}\right)$. A schematic drawing of the MiniBooNE detector is shown in Figure 9, while a photograph of the inside of the tank is shown in Figure 10. There are a total of 12808 -inch detector phototubes (covering 10\% of the surface area) and 240 veto phototubes. The fiducial volume has a $5 \mathrm{~m}$ radius and corresponds to approximately 450 tons. A comparison between the LSND and MiniBooNE experiments is given in Table 1.

3.3. Oscillation Results. Figure 11 shows the estimated neutrino fluxes for neutrino mode and antineutrino mode, respectively. The fluxes are fairly similar (the intrinsic electron-neutrino background is approximately $0.6 \%$ for both modes of running), although the wrong-sign contribution to the flux in antineutrino mode $(\sim 18 \%)$ is much larger than in neutrino mode $(\sim 6 \%)$. The average $\nu_{e}$ plus $\bar{\nu}_{e}$ energies are $0.96 \mathrm{GeV}$ in neutrino mode and $0.77 \mathrm{GeV}$ in antineutrino mode, while the average $\nu_{\mu}$ plus $\bar{\nu}_{\mu}$ energies are $0.79 \mathrm{GeV}$ in neutrino mode and $0.66 \mathrm{GeV}$ in antineutrino mode. The estimated backgrounds in the two modes are very similar, especially at low energy.

Figure 12 shows the reconstructed neutrino and antineutrino energy distributions, $E_{\nu}^{Q E}$, for candidate $\nu_{e}$ and $\bar{v}_{e}$

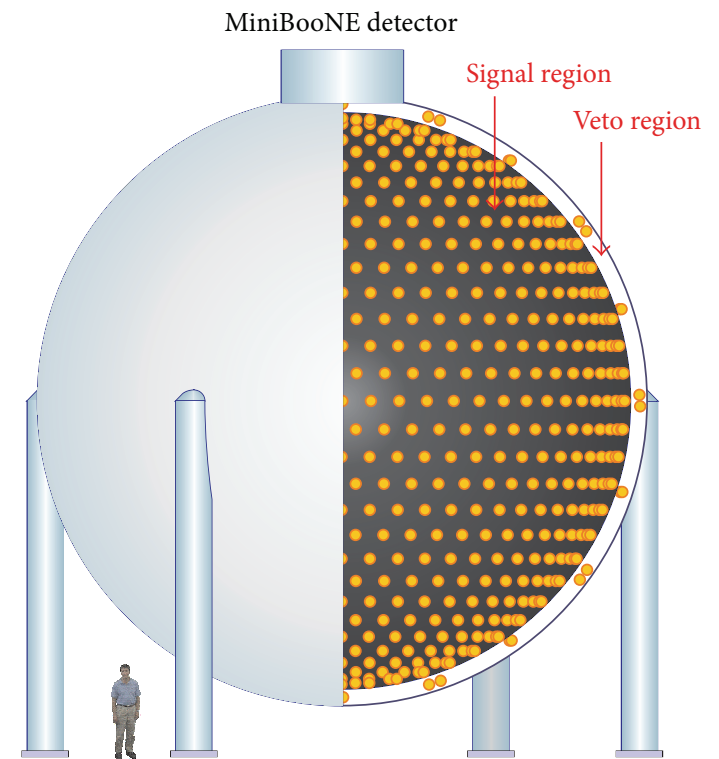

Figure 9: A schematic drawing of the MiniBooNE detector.

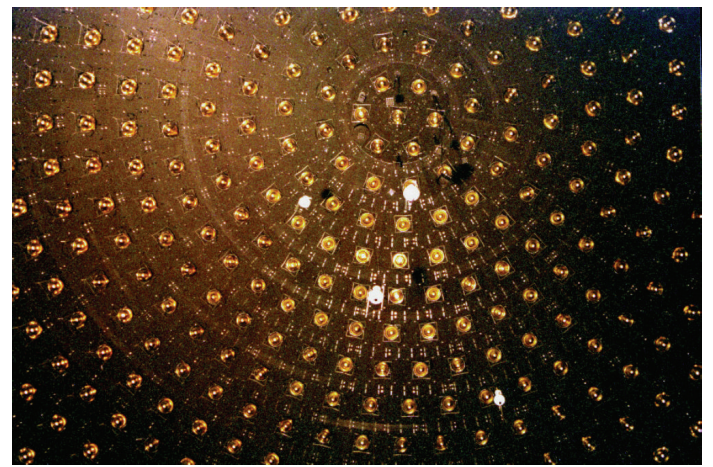

FIGURE 10: A photograph of the inside of the MiniBooNE detector.

data events (points with error bars) compared to the MC simulation (histogram) [7-9], while Figure 13 shows the event excesses in neutrino and antineutrino mode as a function of $E_{\gamma}^{\mathrm{QE}} . E_{\nu}^{\mathrm{QE}}$ assumes that the events are due to chargedcurrent quasi-elastic scattering. The magnitude and energy shape of the excess in antineutrino mode are very similar to what is expected from neutrino oscillations based on the LSND signal, as shown by the pink and green LSND reference 


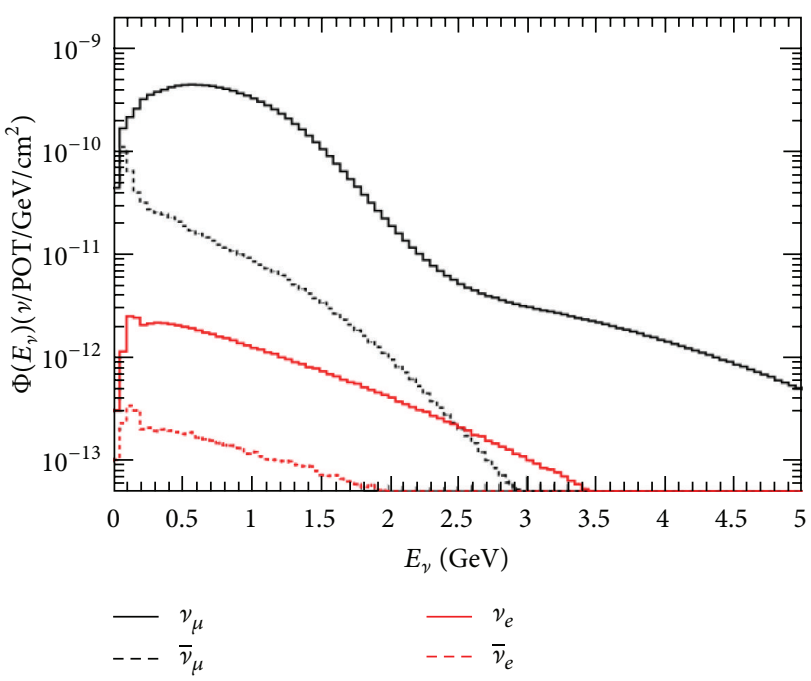

(a)

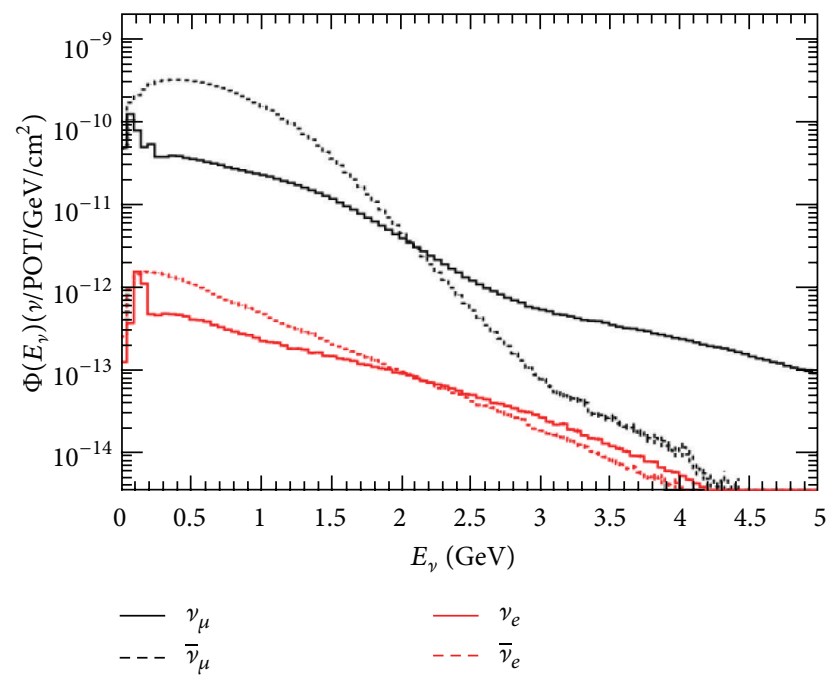

(b)

FIgURE 11: The estimated MiniBooNE neutrino fluxes for neutrino mode (a) and antineutrino mode (b).

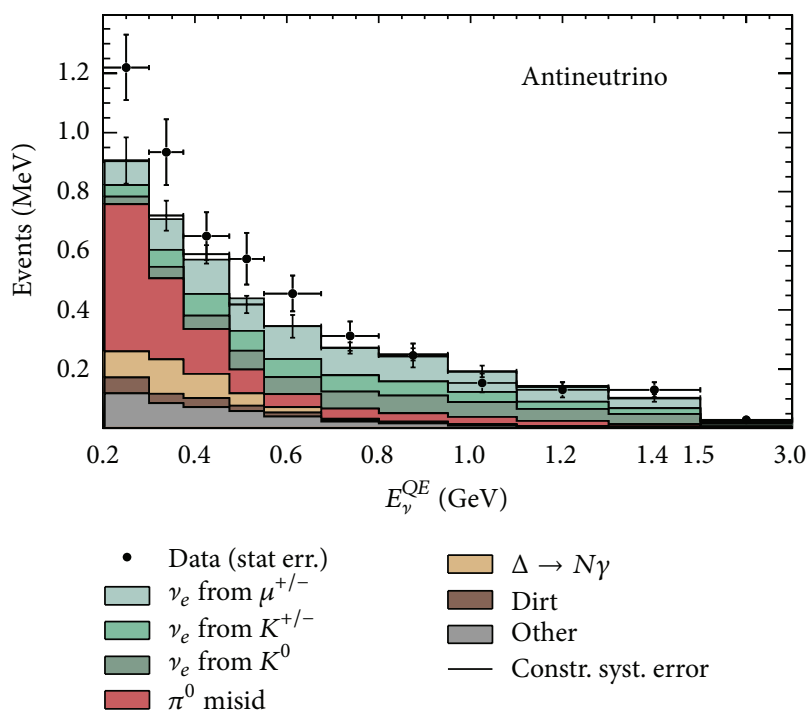

(a)

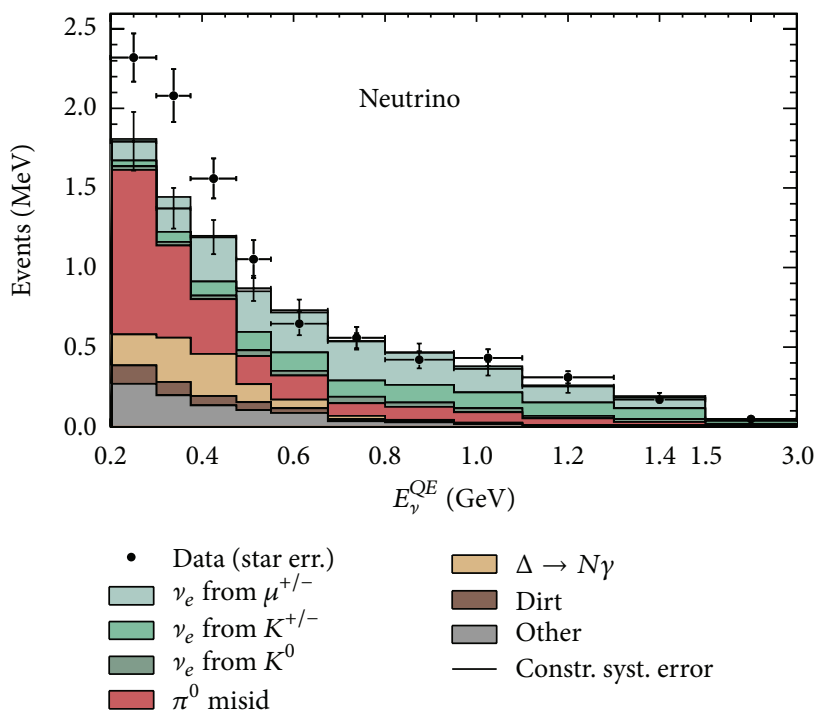

(b)

FIgURE 12: The MiniBooNE neutrino mode (a) and antineutrino mode (b) neutrino energy distributions for $v_{e}$ charged-current quasi-elastic data (points with statistical errors) and background (histogram with systematic errors).

histograms. In neutrino mode, the magnitude of the excess is consistent with LSND; however, the energy shape is softer than the pink and green LSND reference histograms.

Table 4 shows the number of data, background, and excess events for neutrino mode, antineutrino mode, and combined in the neutrino oscillation energy range from 200 to $1250 \mathrm{MeV}$. The uncertainties include both statistical and constrained systematic errors, where the $v_{m} u$ chargedcurrent data sample is used to normalize the backgrounds. All known systematic errors are included in the systematic error estimate. In neutrino mode, an excess of $162.0 \pm$ 47.8 events, corresponding to $3.4 \sigma$, is observed, while in antineutrino mode, the observed excess is $78.4 \pm 28.5$ events, corresponding to $2.8 \sigma$. Combining the data in neutrino and antineutrino modes, the total excess is $240.3 \pm 62.9$ events, corresponding to $3.8 \sigma$.

Figure 14 shows the MiniBooNE oscillation allowed regions [7-9] from fits to the antineutrino data (a) and neutrino data (b). In antineutrino mode, the probability of the best fit is good (66\%, corresponding to a $\left.\chi^{2} / \mathrm{ndf}=5.0 / 7.0\right)$, and the MiniBooNE allowed region overlaps well with the LSND allowed region for $\Delta m^{2}<1 \mathrm{eV}^{2}$, while the KARMEN experiment [26] rules out most of the LSND allowed region for $\Delta m^{2}>2 \mathrm{eV}^{2}$. Therefore, the MiniBooNE antineutrino data are consistent with simple two-neutrino oscillations and 


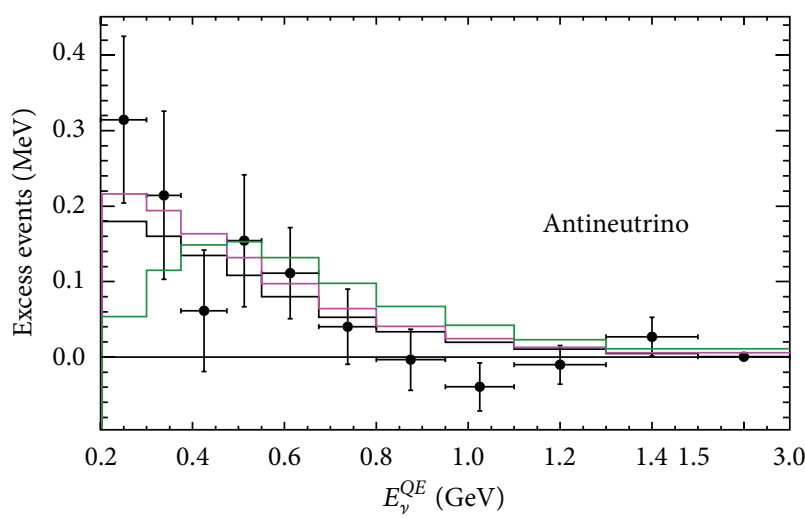

- Data-expected background

$-\sin ^{2} 2 \theta=0.004, \Delta m^{2}=1.0 \mathrm{eV}^{2}$

$-\sin ^{2} 2 \theta=0.2, \Delta m^{2}=0.1 \mathrm{eV}^{2}$

— MiniBooNE $2 v$ best fit

(a)

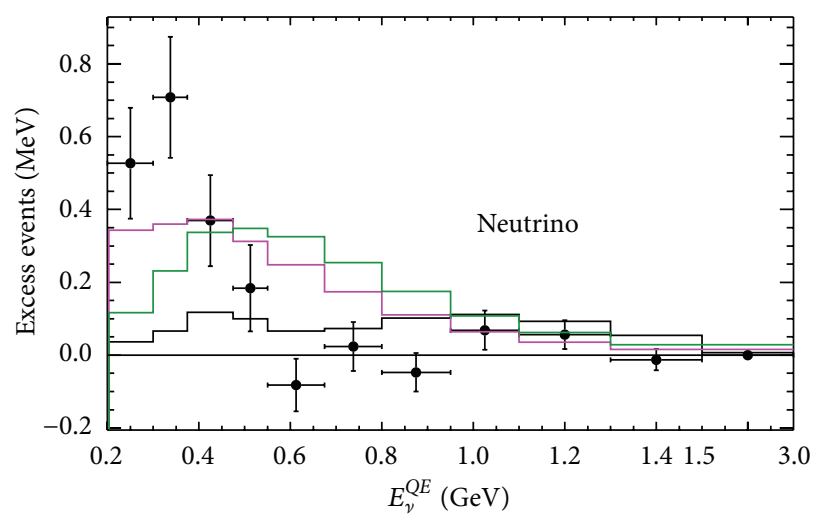

- Data-expected background

$\sin ^{2} 2 \theta=0.004, \Delta m^{2}=1.0 \mathrm{eV}^{2}$

$-\sin ^{2} 2 \theta=0.2, \Delta m^{2}=0.1 \mathrm{eV}^{2}$

- MiniBooNE $2 v$ best fit

(b)

Figure 13: The MiniBooNE neutrino mode (a) and antineutrino mode (b) event excesses as a function of neutrino energy. Also the expectations from the best two-neutrino oscillation fits with neutrino energy from 200 to $3000 \mathrm{MeV}$ and from two reference values in the LSND allowed region are shown. All known systematic errors are included in the systematic error estimate.

TABLE 4: The number of data, fitted (constrained) backgrounds, and excess events in the $\nu_{e}$ and $\bar{\nu}_{e}$ analyses for neutrino mode, antineutrino mode, and combined in the neutrino oscillation energy range from 200 to $1250 \mathrm{MeV}$. The uncertainties include both statistical and constrained systematic errors. All known systematic errors are included in the systematic error estimate.

\begin{tabular}{lccc}
\hline Mode & Data & Background & Excess \\
\hline Neutrino mode & 952 & $790.0 \pm 28.1 \pm 38.7$ & $162.0 \pm 47.8$ \\
Antineutrino mode & 478 & $399.6 \pm 20.0 \pm 20.3$ & $78.4 \pm 28.5$ \\
Combined & 1430 & $1189.7 \pm 34.5 \pm 52.6$ & $240.3 \pm 62.9$ \\
\hline
\end{tabular}

with the LSND oscillation signal. In neutrino mode, the probability of the best fit is not as good $(6.1 \%$, corresponding to a $\left.\chi^{2} / \mathrm{ndf}=13.2 / 6,8\right)$, and the MiniBooNE allowed region overlaps a smaller fraction of the LSND allowed region at low $\Delta m^{2}$, which is already ruled out by the ICARUS experiment [30]. Therefore, in neutrino mode, the data are marginally compatible with a simple two-neutrino oscillation formalism. Indeed, the initial MiniBooNE oscillation publication [34] showed that there is hardly any excess of events above $475 \mathrm{MeV}$ in neutrino mode, ruling out simple two-neutrino oscillations as an explanation of the LSND signal. However, expanded models with several sterile neutrinos can reduce the incompatibility by allowing for $\mathrm{CP}$ violating effects between neutrino and antineutrino oscillations [10, 11].

Figure 15 shows the MiniBooNE oscillation allowed regions from a combined fit to the antineutrino plus neutrino data samples [35]. As in neutrino mode, the probability of the best fit is not very good (6.7\%). However, as discussed above, expanded models with several sterile neutrinos can improve the fit probability by allowing for $\mathrm{CP}$ violating effects between neutrino and antineutrino oscillations $[10,11]$.

\section{Global Neutrino Oscillation Analyses}

Several global $3+N$ oscillation analyses have been performed on the world neutrino data that include both long-baseline and short-baseline oscillation experiments, including both $\nu_{\mu} \rightarrow \nu_{e}$ and $\bar{\nu}_{\mu} \rightarrow \bar{\nu}_{e}$ appearance data and $\nu_{\mu}$ and $\bar{v}_{\mu}$ disappearance data [12]. In general, the analyses assume a model with 3 light, mostly active neutrinos, $N$ heavier, mostly sterile neutrinos, and $3+N$ neutrino mass eigenstates. Precision measurements of the $Z^{\circ}$ width have determined that the number of active neutrinos is approximately 3 $(2.984 \pm 0.008$ [19]), so that additional neutrinos beyond the three active neutrinos would need to be sterile to the weak interaction. Note that neutrino oscillations depend on the superposition of neutrino mass eigenstates and do not depend on whether neutrinos are active or sterile, so that the $3+N$ neutrino mass eigenstates would result in $2+N$ independent $\Delta m^{2}$ values. Note also that, for $N>1$, there would exist $\mathrm{CP}$ violation parameters for both short-baseline and long-baseline experiments. Finally, note that, for $3+N$ models, neutrinos in general would be a superposition of both active and sterile components.

Table 5 shows the best fit values from recent $3+1$ global fits to the world neutrino data from $[10,11]$. (Additional global fits can be found in [12].) Reference [10] includes data from the LSND, MiniBooNE, KARMEN, NOMAD, CCFR, CDHS, MINOS, Bugey, SAGE, and GALLEX experiments, while [11] also includes data from the E776 and ICARUS experiments. For both global fits, the best fit value of $\Delta m_{41}^{2}$ is $\sim 0.9 \mathrm{eV}^{2}$ and the $\chi^{2}$ goodness of fit probabilities (GOF) 


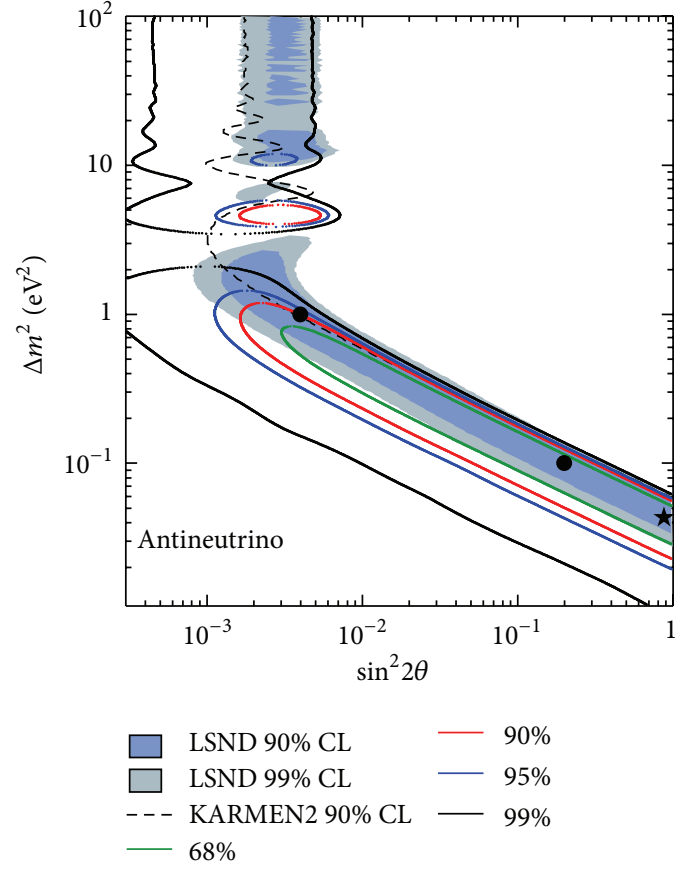

(a)

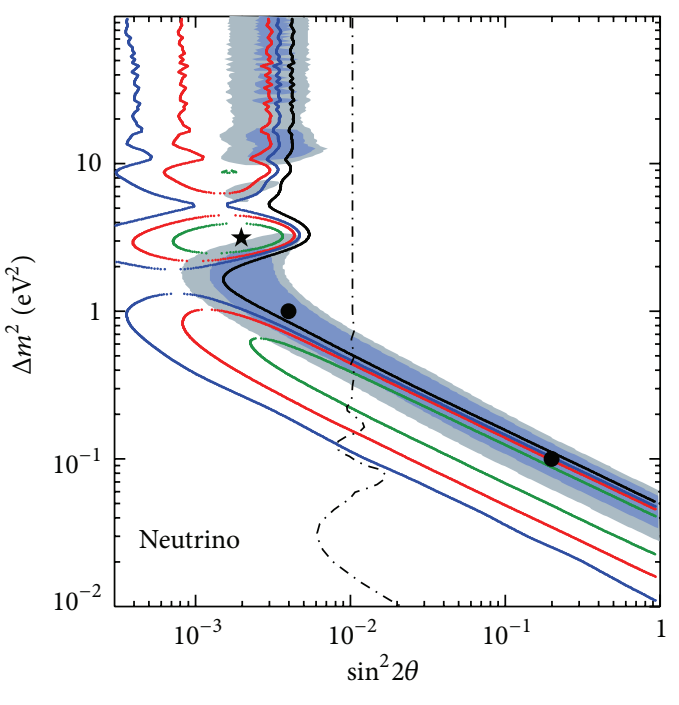

-.. ICARUS $90 \%$ CL

(b)

FIGURE 14: MiniBooNE allowed regions in antineutrino mode (a) and neutrino mode (b) for events with neutrino energy greater than $200 \mathrm{MeV}$ within a two-neutrino oscillation model. Also the ICARUS [30] and KARMEN [26] appearance limits for neutrinos and antineutrinos are shown, respectively. The shaded areas show the 90\% and 99\% CL LSND $\bar{\nu}_{\mu} \rightarrow \bar{\nu}_{e}$ allowed regions. The black stars show the MiniBooNE best fit points, while the circles show two reference values in the LSND allowed region.

are reasonable at $55 \%$ and $19 \%$, respectively. However, the parameter goodness of fit probabilities (PGOF) are very low at $0.043 \%$ and $0.012 \%$, respectively. The PGOF [36] is based on the difference between the overall best fit chi square and the sum of the best fit chi squares for the appearance-only and disappearance-only experiments. Although there is tension between the appearance and disappearance experiments, we believe that fake data studies would need to be performed in order to calculate the PGOF true probabilities [37].

Table 6 shows the best fit values from recent $3+2$ global fits to the world neutrino data from $[10,11]$. (Additional global fits can be found in [12].) The GOF probabilities are improved from the $3+1$ fits, although the PGOF probabilities are worse. As mentioned above, we believe that fake data studies would need to be performed in order to calculate the PGOF true probabilities [37]. Figures 16 and 17 show the $3+2$ best fit $\Delta m_{41}^{2}$ versus $\Delta m_{51}^{2}$ values for the two global fits. Figure 17 also shows the $1+3+1$ best fit, where the two mostly sterile neutrinos have masses less than and greater than the masses of the mostly active neutrinos.

\section{Future Short-Baseline Accelerator Neutrino Experiments}

Future accelerator neutrino experiments will have the capability of testing the current evidence for sterile neutrinos.
TABle 5: The best fit values from $3+1$ global fits to the world neutrino data from $[10,11]$. Also the goodness of fit probability (GOF) and the parameter goodness of fit probability (PGOF) [36] are shown.

\begin{tabular}{cccccc}
\hline Ref. & $\Delta m_{41}^{2}\left(\mathrm{eV}^{2}\right)$ & $\left|U_{e 4}\right|$ & $\left|U_{\mu 4}\right|$ & GOF & PGOF \\
\hline$[10]$ & 0.92 & 0.15 & 0.17 & $55 \%$ & $0.043 \%$ \\
{$[11]$} & 0.93 & 0.15 & 0.17 & $19 \%$ & $0.012 \%$ \\
\hline
\end{tabular}

At Fermilab, MINOS+ and MicroBooNE provide a wellcoordinated program of experiments that should help determine in the near future whether or not sterile neutrinos can explain the existing short-baseline neutrino anomalies. MINOS+ involves running the MINOS detectors with the NuMI beam in medium energy mode for the NoVA experiment, which provides a much higher flux of neutrinos above a few GeV. High $\Delta m^{2} v_{\mu}$ disappearance into sterile neutrinos can be searched for by comparing the event rates in the near and far detectors at higher energies, where the effect of neutrino oscillations at the atmospheric scale is small [38]. MINOS+ should begin taking data later in 2013. The MicroBooNE experiment is under construction in the Booster Neutrino Beam (BNB) just upstream of MiniBooNE at a distance of $0.47 \mathrm{~km}$ from the neutrino source. MicroBooNE [39] consists of a 170-ton (86-ton active volume) liquid argon TPC, that will be able to determine whether the $3.8 \sigma$ excess of events observed by MiniBooNE is due to electron events, as expected for $v_{\mu} \rightarrow v_{e}$ oscillations, or to photon 
TABLE 6: The best fit values from $3+2$ global fits to the world neutrino data from $[10,11]$. Also the goodness of fit probability (GOF) and the parameter goodness of fit probability (PGOF) are shown.

\begin{tabular}{lcccccccrr}
\hline Ref. & $\begin{array}{l}\Delta m_{41}^{2} \\
\left(\mathrm{eV}^{2}\right)\end{array}$ & $\begin{array}{l}\Delta m_{51}^{2} \\
\left(\mathrm{eV}^{2}\right)\end{array}$ & $\left|U_{e 4}\right|$ & $\left|U_{\mu 4}\right|$ & $\left|U_{e 5}\right|$ & $\left|U_{\mu 5}\right|$ & $\phi_{\mathrm{CP}}$ & GOF & PGOF \\
\hline$[10]$ & 0.92 & 17 & 0.15 & 0.17 & 0.069 & 0.16 & $1.8 \pi$ & $69 \%$ \\
{$[11]$} & 0.47 & 0.87 & 0.13 & 0.15 & 0.14 & 0.13 & $-0.15 \pi$ & $23 \%$ & $0.0082 \%$ \\
\hline
\end{tabular}
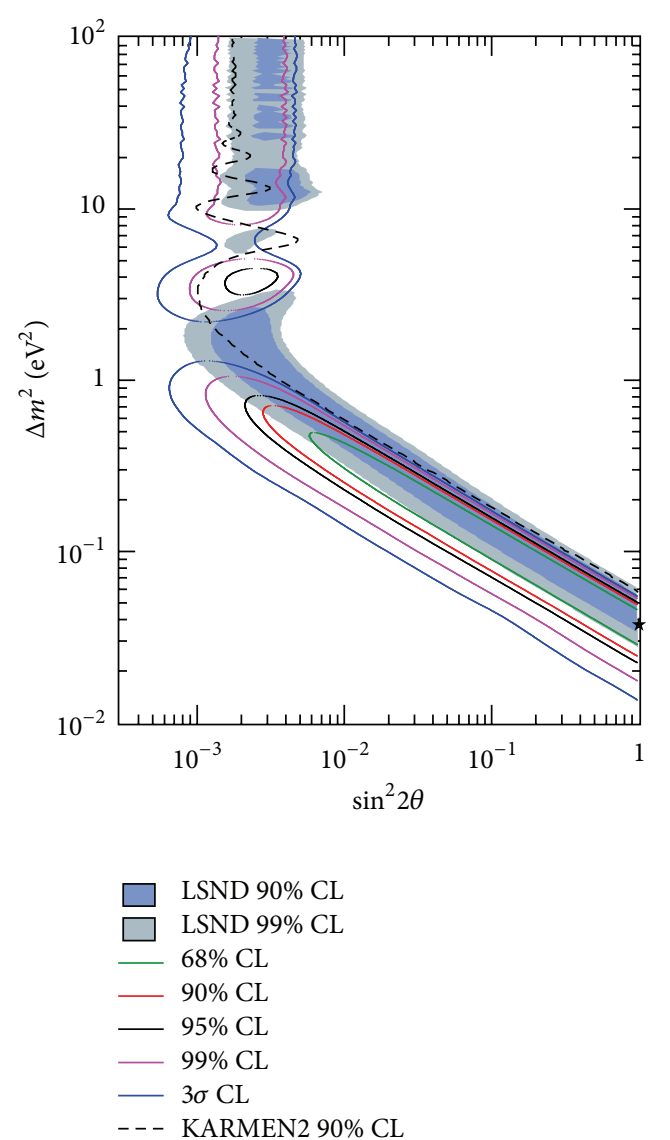

FIGURE 15: MiniBooNE allowed regions in combined neutrino and antineutrino mode for events with neutrino energy from 200 to $3000 \mathrm{MeV}$ within a two-neutrino $\nu_{\mu} \rightarrow \nu_{e}$ and $\bar{\nu}_{\mu} \rightarrow \bar{\nu}_{e}$ oscillation model. Also shown is the $\bar{v}_{\mu} \rightarrow \bar{v}_{e}$ limit from the KARMEN experiment [26]. The shaded areas show the $90 \%$ and $99 \%$ CL LSND $\bar{\nu}_{\mu} \rightarrow \bar{\nu}_{e}$ allowed regions. The black star shows the best fit point.

events, which would indicate some other process. Figure 18 shows the MicroBooNE sensitivity to $\nu_{\mu} \rightarrow v_{e}$ oscillations. MicroBooNE should begin taking data in 2014.

NuSTORM [31] is a far future proposal that involves building a muon storage ring with $\mu^{ \pm}$central momentum of $3.8 \mathrm{GeV} / \mathrm{c}$, and it can be built at either Fermilab or CERN. NuSTORM produces fluxes of neutrinos with wellunderstood energy spectra: $\nu_{\mu}$ and $\bar{\nu}_{e}$ for a $\mu^{-}$stored beam and $\bar{\nu}_{\mu}$ and $\nu_{e}$ for a stored $\mu^{+}$beam. Two magnetized iron neutrino detectors will be built downstream of the storage ring at distances of $\sim 30 \mathrm{~m}$ and $\sim 1500 \mathrm{~m}$ in order to search for $v_{e} \rightarrow v_{\mu}$ appearance and $\nu_{e}$ and $\nu_{\mu}$ disappearance. The

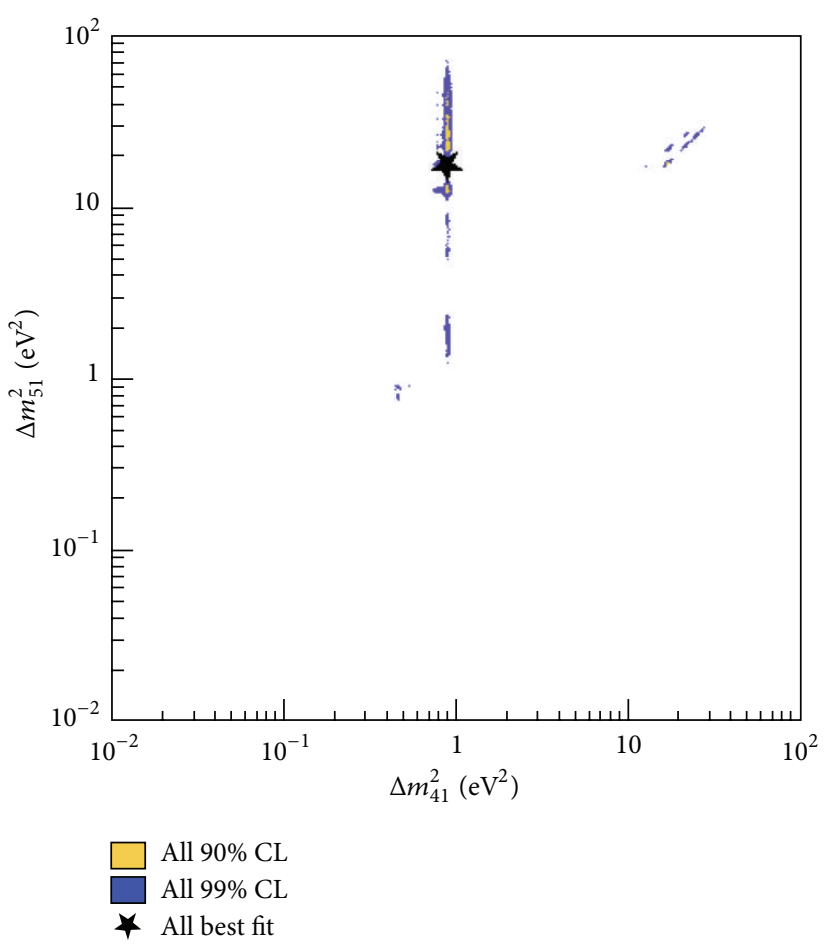

FIGURE 16: The $3+2$ best fit $\Delta m_{41}^{2}$ versus $\Delta m_{51}^{2}$ values from [10].

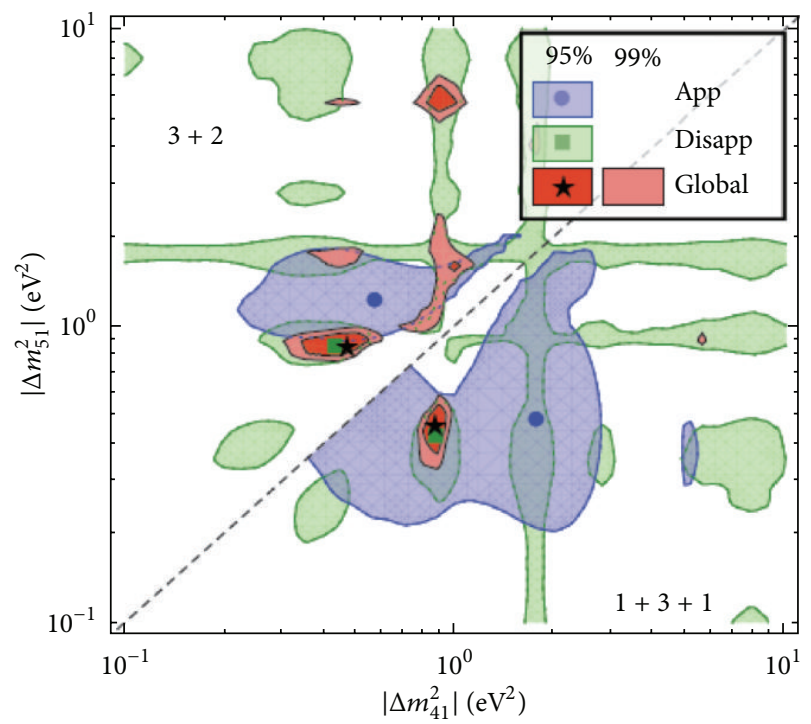

FIGURE 17: The $3+2$ and $1+3+1$ best fit $\Delta m_{41}^{2}$ versus $\Delta m_{51}^{2}$ values from [11]. 


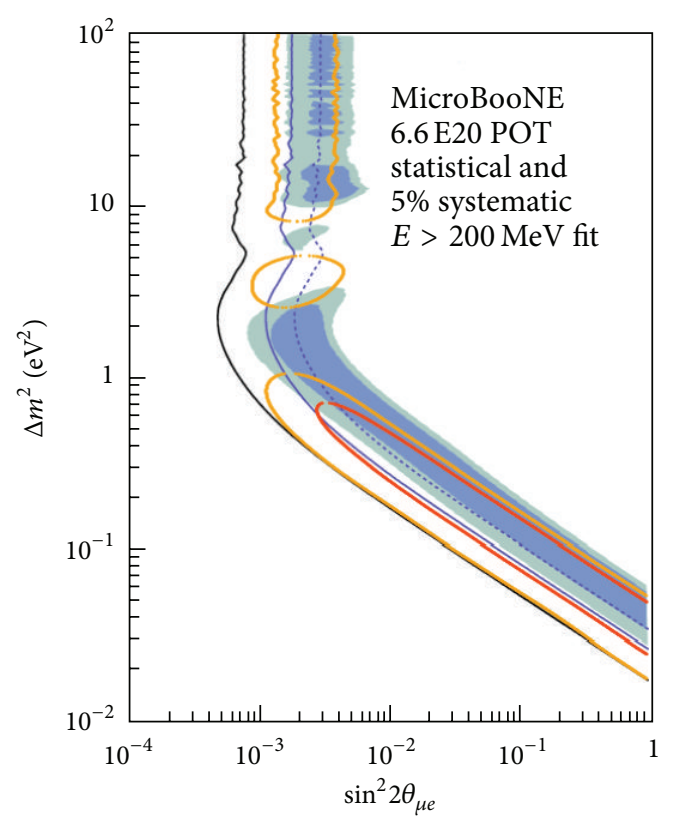

$\begin{array}{ll}\square & \text { LSND 90\% CL } \\ \square & \text { LSND 99\% CL } \\ - & \text { MiniBooNE 90\% CL } \\ - & \text { MiniBooNE 99\% CL } \\ - & 90 \% \mathrm{CL} \\ - & 3 \sigma \mathrm{CL} \\ \ldots . . . & 5 \sigma \mathrm{CL}\end{array}$

FIgure 18: The MicroBooNE sensitivity to $\nu_{\mu} \rightarrow v_{e}$ oscillations. Also the allowed regions from LSND and from the MiniBooNE combined fit are shown.

allowed LSND and MiniBooNE oscillation regions can be covered at the $10 \sigma$ level, as shown in Figure 19.

ICARUS-NESSIE [32] is another far future proposal for an experiment at CERN to test the LSND and MiniBooNE oscillation signals. A new neutrino beamline would be built from the SPS with both near and far liquid argon TPC detectors (ICARUS) and muon spectrometers (NESSIE). The near detector location would be at a distance of $300 \mathrm{~m}$ from the neutrino source and would contain a 150-ton (T150) liquid argon TPC followed by a muon spectrometer with a dipole magnetic field and iron slabs. The far detector location would be at a distance of $1600 \mathrm{~m}$ from the neutrino source and would contain a 600-ton LAr TPC (T600) followed by a muon spectrometer. ICARUS-NESSIE will be able to search for both $v_{\mu}$ disappearance and $v_{e}$ appearance. Figure 20 shows the expected sensitivities for $v_{\mu} \rightarrow v_{e}$ oscillations (a) and $\bar{v}_{\mu} \rightarrow \bar{\nu}_{e}$ oscillations (b) after one year and two years of data taking, respectively. ICARUS-NESSIE will fully cover the LSND and MiniBooNE oscillation allowed regions.

The OscSNS and IsoDAR proposals offer the unique opportunity to observe actual oscillations in the detector (i.e., an oscillation of the number of events in the detector as a function of distance from the neutrino source, correcting for the $1 / r^{2}$ falloff of the neutrino flux), which would be unmistakable proof for short baseline neutrino oscillations. The OscSNS proposal [40] involves building a cylindrical

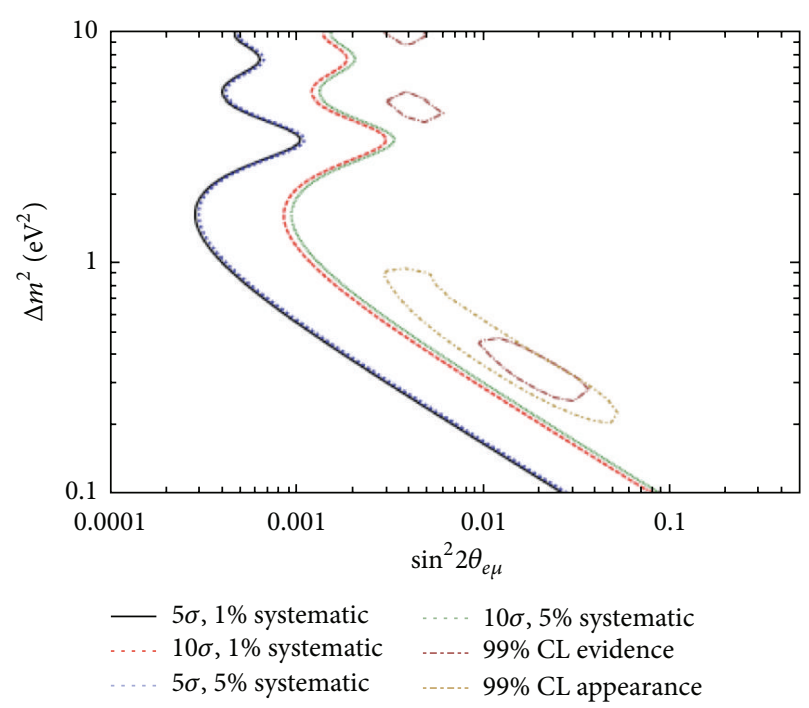

FIGURE 19: NuSTORM can cover the allowed LSND and MiniBooNE oscillation regions at the $10 \sigma$ level (from [31]).

detector approximately $60 \mathrm{~m}$ from the SNS spallation target [41]. The 1.4 MW beam power of the SNS is a prodigious source of neutrinos from the decay of $\pi^{+}$and $\mu^{+}$at rest. These decays produce a well specified flux of neutrinos via $\pi^{+} \rightarrow \mu^{+} \nu_{\mu}, \tau_{\pi}=2.7 \times 10^{-8} \mathrm{~s}$ and $\mu^{+} \rightarrow e^{+} \nu_{e} \bar{\nu}_{\mu}, \tau_{\mu}=$ $2.2 \times 10^{-6} \mathrm{~s}$. The low duty factor of the SNS ( $\sim 695 \mathrm{~ns}$ beam pulses at $\left.60 \mathrm{~Hz}, \mathrm{DF}=4.2 \times 10^{-5}\right)$ is more than 1000 times less than that found at LAMPF. This smaller duty factor provides a reduction in backgrounds due to cosmic rays, and allows the $v_{\mu}$ induced events from $\pi^{+}$decay to be separated from the $\nu_{e}$ and $\bar{\nu}_{\mu}$ induced events from $\mu^{+}$decay. The OscSNS detector will be centered at a location 60 meters from the SNS target, in the backward direction. The cylindrical detector design is based upon the LSND and MiniBooNE detectors and will consist of an 800-ton tank of mineral oil (with a small concentration of b-PBD scintillator dissolved in the oil) that is covered by approximately 35008 -inch phototubes, yielding a photocathode coverage of $25 \%$. Figure 21 shows the expected sensitivity for $\bar{\nu}_{e}$ appearance after two and six calendar years of run time. The LSND allowed region is fully covered by more than $5 \sigma$.

The IsoDAR proposal [33] involves the construction of a high-intensity proton cyclotron near an underground detector to search for $\bar{\nu}_{e}$ disappearance. The proposed cyclotron would accelerate $\mathrm{H}_{2}^{+}$ions up to $60 \mathrm{MeV} / \mathrm{amu}$ at an intensity of $5 \mathrm{~mA}$. The $60 \mathrm{MeV}$ proton beam then interacts on a ${ }^{9} \mathrm{Be}$ target that is surrounded by a ${ }^{7} \mathrm{Li}$ sleeve. Neutrons produced in the Be target interact in the sleeve to produce ${ }^{8} \mathrm{Li}$, which decay to produce $\bar{v}_{e}$ with an average energy of $6.4 \mathrm{MeV}$. The $\bar{\nu}_{e}$ can then interact in a kiloton underground detector, such as KamLAND [42] in Japan. By fitting for oscillations in the detector, IsoDAR would have excellent sensitivity to $\bar{v}_{e}$ disappearance, as shown in Figure 22. 


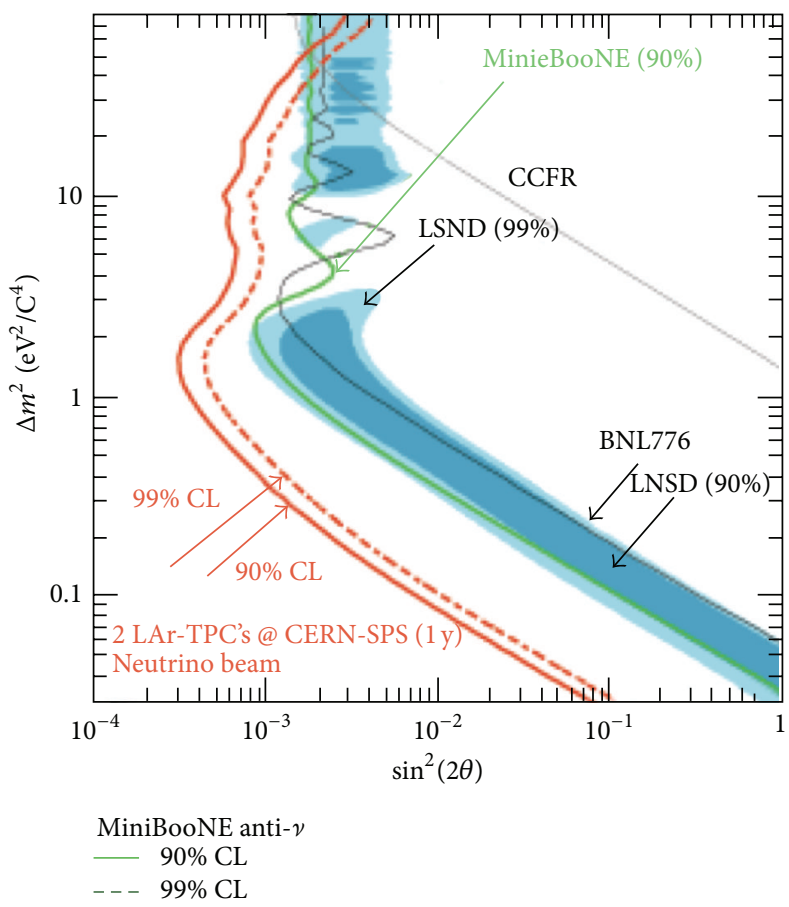

(a)

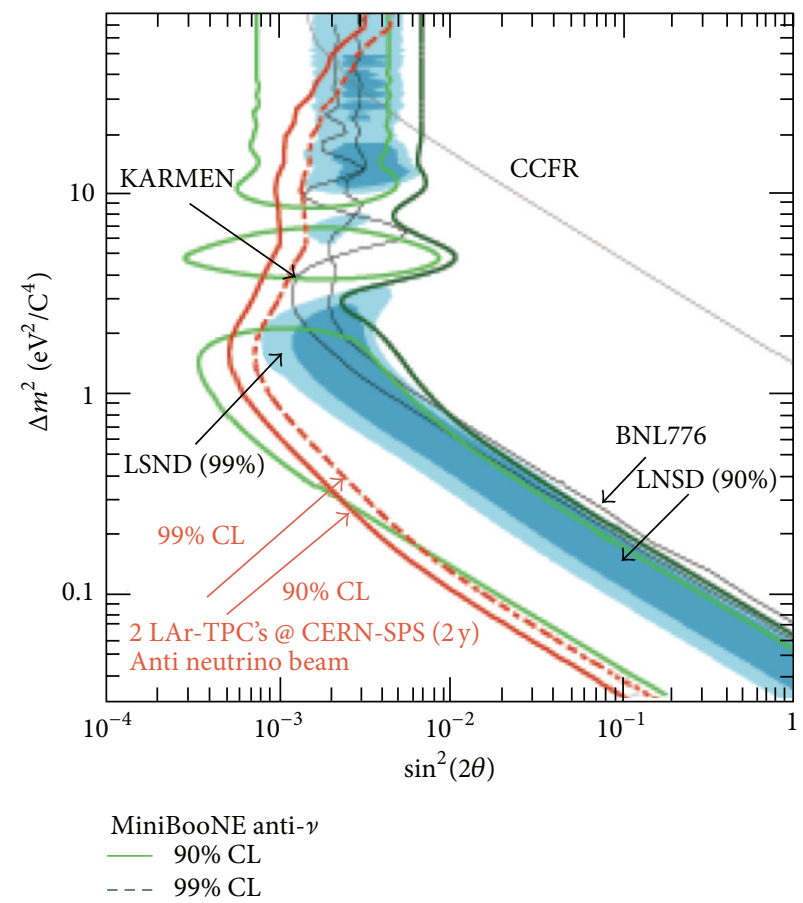

(b)

FIGURE 20: The ICARUS-NESSIE expected sensitivities for $\nu_{\mu} \rightarrow \nu_{e}$ oscillations (a) and $\bar{\nu}_{\mu} \rightarrow \bar{\nu}_{e}$ oscillations (b) after one year and two years of data taking, respectively (from [32]).

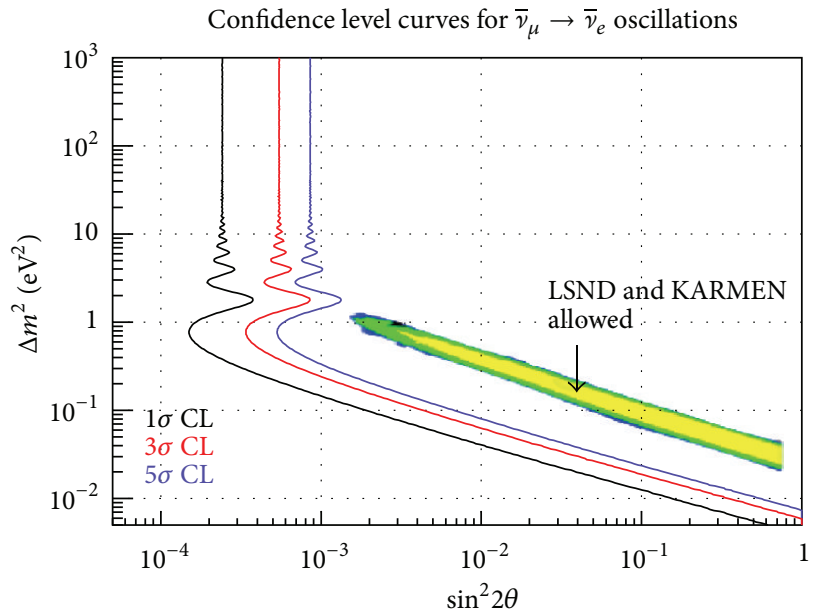

(a)

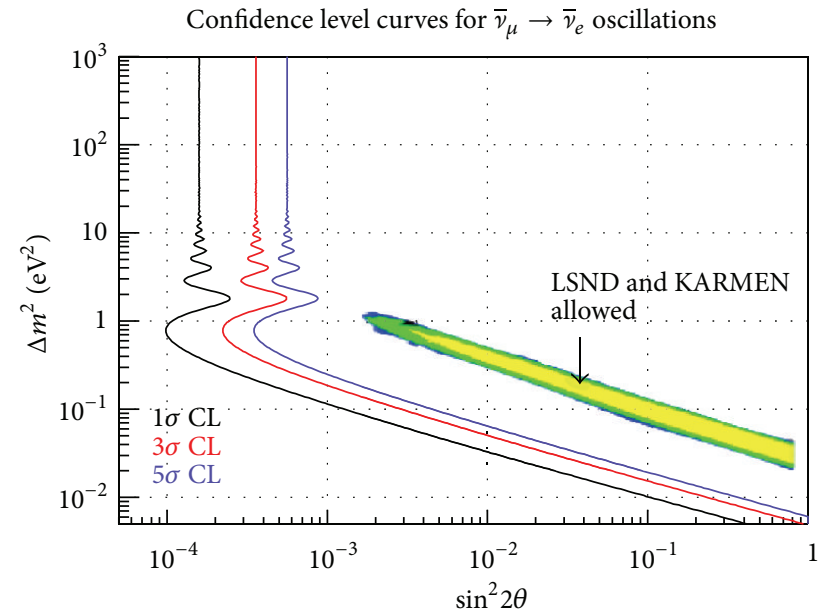

(b)

Figure 21: The OscSNS sensitivity curves for the simulated sensitivity to $\bar{v}_{\mu} \rightarrow \bar{v}_{e}$ oscillations after two (a) and six (b) calendar years of operation. Note that OscSNS has more than $5 \sigma$ sensitivity to the LSND allowed region after 2 years of data collection.

\section{Conclusions}

The LSND and MiniBooNE experiments both observe excesses of $\nu_{e}$ candidate events at the $3.8 \sigma$ level. Combining these two experiments results in a total excess that is over $5 \sigma$ in significance. Whereas no standard model explanation for these excesses has yet been found, $3+N$ oscillation models with 3 light, mostly active neutrinos and $N$ heavy, mostly sterile neutrinos provide a fairly good fit to the world neutrino oscillation data, although there is some tension at present between appearance and disappearance oscillation experiments. Future experiments at Fermilab, CERN, ORNL, and in Japan will test this neutrino oscillation evidence and have the potential to prove the existence of light, sterile neutrinos. Such sterile neutrinos would have a big impact on particle physics, nuclear physics, and astrophysics. 


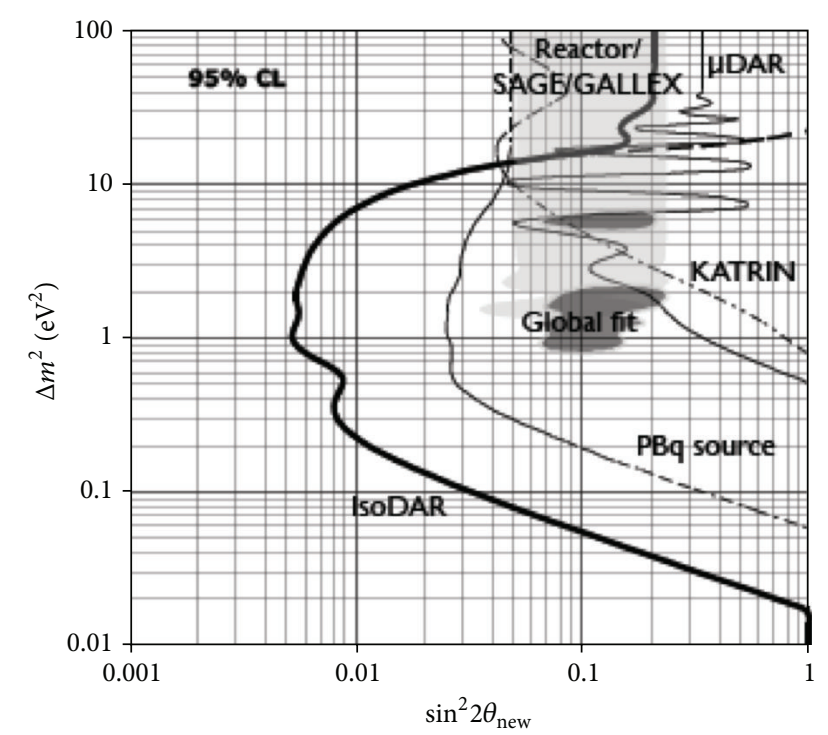

Figure 22: The sensitivity of the IsoDAR experiment to $\bar{\nu}_{e}$ disappearance in a five-year physics run (from [33]).

\section{Acknowledgments}

The author thanks the Department of Energy for support. This work was made possible by the dedicated efforts of the LSND and MiniBooNE collaborations.

\section{References}

[1] G. L. Fogli, E. Lisi, A. Marrone, D. Montanino, A. Palazzo, and A. M. Rotunno, "Addendum to 'Solar neutrino oscillation parameters after first KamLAND results,' Physical Review D, vol. 69, no. 1, Article ID 017301, 2004.

[2] C. Athanassopoulos, L. B. Auerbach, D. A. Bauer et al., "Candidate events in a search for $\bar{v}_{\mu} \rightarrow \bar{v}_{e}$ oscillations," Physical Review Letters, vol. 75, no. 14, pp. 2650-2653, 1995.

[3] C. Athanassopoulos, L. B. Auerbach, R. L. Burman et al., "Evidence for $\bar{v}_{\mu} \rightarrow \bar{v}_{e}$ oscillations from the LSND experiment at the los alamos meson physics facility," Physical Review Letters, vol. 77, pp. 3082-3085, 1996.

[4] C. Athanassopoulos, L. B. Auerbach, R. L. Burman et al., "Results on $v_{\mu} \rightarrow v_{e}$ neutrino oscillations from the LSND experiment," Physical Review Letters, vol. 81, pp. 1774-1777, 1998.

[5] C. Athanassopoulos, L. B. Auerbach, R. L. Burman et al., "Results on $v_{\mu} \rightarrow v_{e}$ oscillations from pion decay in flight neutrinos," Physical Review C, vol. 58, pp. 2489-2511, 1998.

[6] A. Aguilar, L. B. Auerbach, R. L. Burman et al., "Evidence for neutrino oscillations from the observation of $\bar{v}_{e}$ appearance in a $\bar{v}_{\mu}$ beam," Physical Review D, vol. 64, no. 11, Article ID 112007, 2001.

[7] A. A. Aguilar-Arevalo, C. E. Anderson, A. O. Bazarko et al., "Unexplained excess of electronlike events from a $1-\mathrm{GeV}$ neutrino beam," Physical Review Letter, vol. 102, no. 10, Article ID 101802, 2009.

[8] A. A. Aguilar-Arevalo, C. E. Anderson, A. O. Bazarko et al., "Event excess in the MiniBooNE search for $\bar{v}_{\mu} \rightarrow \bar{v}_{e}$ oscillations," Physical Review Letter, vol. 105, no. 10, Article ID 181801, 2010.
[9] A. A. Aguilar-Arevalo, C. E. Anderson, A. O. Bazarko et al., "Improved search for $\bar{v}_{\mu} \rightarrow \bar{v}_{e}$ oscillations in the MiniBooNE experiment," Physical Review Letter, vol. 110, no. 10, Article ID 161801, 2013.

[10] J. M. Conrad, C. M. Ignarra, G. Karagiorgi, M. H. Shaevitz, and J. Spitz, "Sterile Neutrino Fits to Short-Baseline Neutrino Oscillation Measurements," Advances in High Energy Physics, vol. 2013, Article ID 163897, 26 pages, 2013.

[11] K. Joachim, A. N. Pedro Machado, M. Michele, and T. Schwetz, "Sterile neutrino oscillations: the global picture," Journal of High Energy Physics, vol. 2013, article 50, 2013.

[12] K. N. Abazajian, M. A. Acero, S. K. Agarwalla et al., "Light sterile neutrinos: a white paper," http://arxiv.org/abs/1204.5379.

[13] P. Langacker, The Standard Model and Beyond, Taylor and Francis, Boca Raton, Fla, USA, 2010.

[14] A. de Gouvea and W.-C. Huang, "Constraining the (lowenergy) type-I Seesaw," Physical Review D, vol. 85, Article ID 053006, 12 pages, 2012.

[15] V. A. Kostelecky and M. Mewes, "Lorentz and CPT violation in neutrinos," Physical Review D, vol. 69, no. 1, Article ID 016005, 2004.

[16] T. Katori, V. A. Kostelecky, and R. Tayloe, "Global threeparameter model for neutrino oscillations using Lorentz violation," Physical Review D, vol. 74, Article ID 105009, 2006.

[17] S. N. Gninenko, "MiniBooNE anomaly and heavy neutrino decay," Physical Review Letter, vol. 103, no. 24, Article ID 241802, 2009.

[18] S. N. Gninenko and D. S. Gorbunov, "MiniBooNE anomaly, the decay $D_{s}^{+} \rightarrow \mu^{+} \nu_{\mu}$ and heavy sterile neutrino," Physical Review $D$, vol. 81, no. 7, Article ID 075013, 2010.

[19] ALEPH Collaboration, DELPHI Collaboration, L3 Collaboration, OPAL Collaboration, and SLD Collaboration, "Precision electroweak measurements on the $\mathrm{Z}$ resonance," Physical Report, vol. 427, no. 5-6, p. 257, 2006.

[20] C. Athanassopoulos, L. B. Auerbach, D. Bauer et al., "The liquid scintillator neutrino detector and LAMPF neutrino source," Nuclear Instruments and Methods in Physics Research A, vol. 388, no. 1-2, pp. 149-172, 1997.

[21] R. L. Burman, M. E. Potter, and E. S. Smith, "Monte Carlo simulation of neutrino production by medium-energy protons in a beam stop," Nuclear Instruments and Methods in Physics Research A, vol. 291, no. 3, pp. 621-633, 1990.

[22] R. L. Burman, A. C. Dodd, and P. Plischke, "Neutrino flux calculations for the ISIS spallation neutron facility," Nuclear Instruments and Methods in Physics Research A, vol. 368, no. 2, pp. 416-424, 1996.

[23] R. A. Reeder, B. D. Dieterle, C. Gregory, F. Schaefer, and K. Schum, "Dilute scintillators for large-volume tracking detectors," Nuclear Instruments and Methods in Physics Research A, vol. 334, pp. 353-366, 1993.

[24] J. J. Napolitano, S. J. Freedman, G. T. Garvey et al., "Construction and performance of a large area liquid scintillator cosmic ray anticoincidence detector," Nuclear Instruments and Methods in Physics Research Section A, vol. 274, no. 1-2, pp. 152-164, 1989.

[25] B. Achkara, R. Aleksanb, M. Aveniera et al., "Search for neutrino oscillations at 15, 40 and 95 meters from a nuclear power reactor at Bugey," Nuclear Physics B, vol. 434, pp. 503-534, 1995.

[26] B. Armbruster, I. M. Blair, B. A. Bodmann et al., "Upper limits for neutrino oscillations $v_{\mu}^{-} \rightarrow v_{e}^{-}$from muon decay at rest," Physical Review D, vol. 65, Article ID 112001, 2002. 
[27] G. Mention, M. Fechner, T. Lasserre et al., "Reactor antineutrino anomaly," Physical Review D, vol. 83, no. 7, Article ID 073006, 2011.

[28] A. A. Aguilar-Arevalo, C. E. Anderson, A. O. Bazarko et al., "Neutrino flux prediction at MiniBooNE," Physical Review D, vol. 79, no. 7, Article ID 072002, 2009.

[29] A.A. Aguilar-Arevaloe, C.E. Andersonp, L.M. Bartoszek et al., "The MiniBooNE detector," Nuclear Instruments and Methods in Physics Research A, vol. 599, no. 1, pp. 28-46, 2009.

[30] M. Antonello, B. Baibussinov, P. Benetti et al., "Experimental search for the LSND anomaly with the ICARUS detector in the CNGS neutrino beam," The European Physical Journal C, vol. 73, Article ID 2345, 2013.

[31] D. Adey, S. K. Agarwalla, C. M. Ankenbrandt et al., "Neutrinos from stored Muons nuSTORM: expression of interest," http://arxiv.org/abs/1305.

[32] M. Antonello, D. Bagliani B, and B. Baibussinov, "Baibussinov Search for, "anomalies" from neutrino and anti-neutrino oscillations at $\Delta_{m^{2}} \sim 1 \mathrm{eV}^{2}$ with muon spectrometers and large LArTPC imaging detectors," http://arxiv.org/abs/1203.3432.

[33] A. Bungau, A. Adelmann, J. R. Alonso et al., "Proposal for an electron antineutrino disappearance search using high-rate ${ }^{8} \mathrm{Li}$ production and decay," Physical Review Letter, vol. 109, no. 14, Article ID 141802, 2012.

[34] A. A. Aguilar-Arevalo, A. O. Bazarko, S. J. Brice et al., "Search for electron neutrino appearance at the $\Delta m^{2} \sim 1 \mathrm{eV}^{2}$ scale," Physical Review Letter, vol. 98, no. 23, Article ID 231801, 2007.

[35] A. A. Aguilar-Arevalo, B. C. Brown, L. Bugel et al., "A $\bar{v}_{\mu} \rightarrow$ $\bar{v}_{e}$ combined to and to oscillation analysis of the MiniBooNE excesses," Physical Review Letters, vol. 110, no. 16, Article ID 161801, 2013.

[36] M. Maltoni, T. Schwetz, M. Tortola, and J. Valle, "Ruling out four-neutrino oscillation interpretations of the LSND anomaly?" Nuclear Physics B, vol. 643, no. 1-3, pp. 321-328, 2002.

[37] M. J. Conrad, C. W. Louis, and H. M. Shaevitz, "The LSND and MiniBooNE oscillation searches at high $\Delta m^{2}$," Annual Review of Nuclear and Particle Science, vol. 63, p. 45, 2013.

[38] P. Adamson, D. J. Auty, D. S. Ayres et al., "Active to sterile neutrino mixing limits from neutral-current interactions in MINOS," Physical Review Letter, vol. 107, no. 1, Article ID 011802, 2011.

[39] G. Karagiorgi, "MicroBooNE: searching for new physics in the neutrino sector with a 100-ton-scale liquid argon TPC," Journal of Physics, vol. 375, Article ID 042067, 2012.

[40] G. T. Garvey, A. Green, C. Green et al., "Measuring active-sterile neutrino oscillations with a stopped pion neutrino source," Physical Review D, vol. 72, no. 9, Article ID 092001, 2005.

[41] "The spallation neutron source (SNS) is an accelerator-based source built in Oak Ridge, Tennessee, by the U.S. DOE," http://sns.gov/.

[42] S. Abe, T. Ebihara, S. Enomoto et al., "Precision measurement of neutrino oscillation parameters with KamLAND," Physical Review Letter, vol. 100, no. 22, Article ID 221803, 5 pages, 2008. 

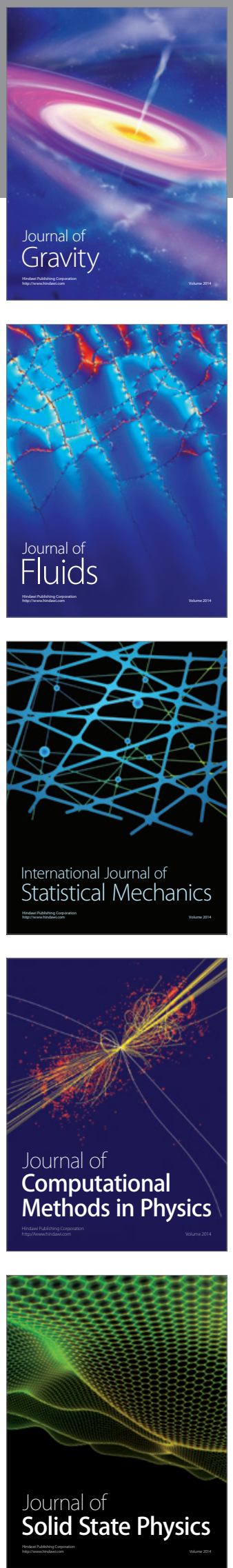

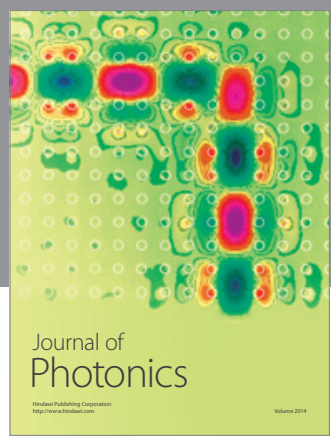

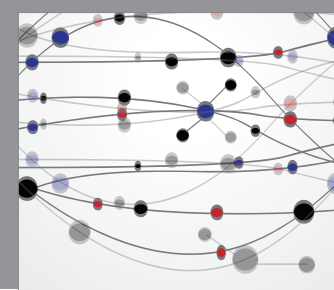

The Scientific World Journal

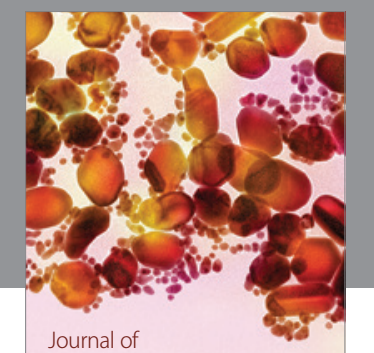

Soft Matter
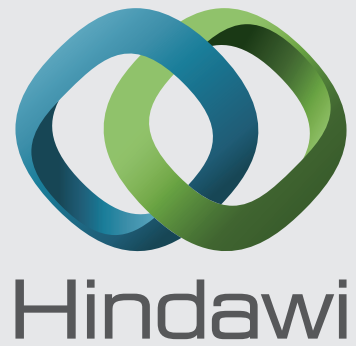

Submit your manuscripts at

http://www.hindawi.com
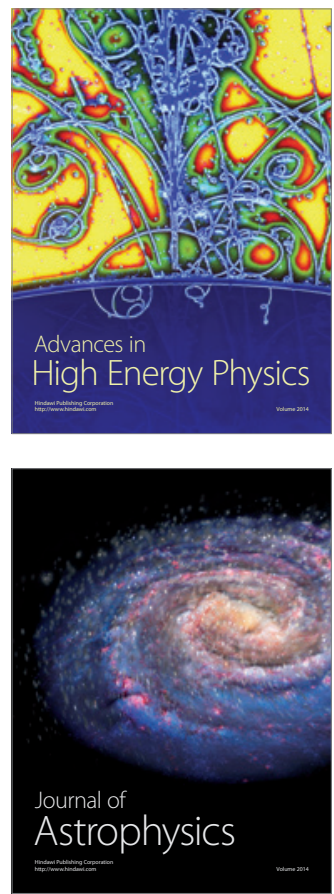
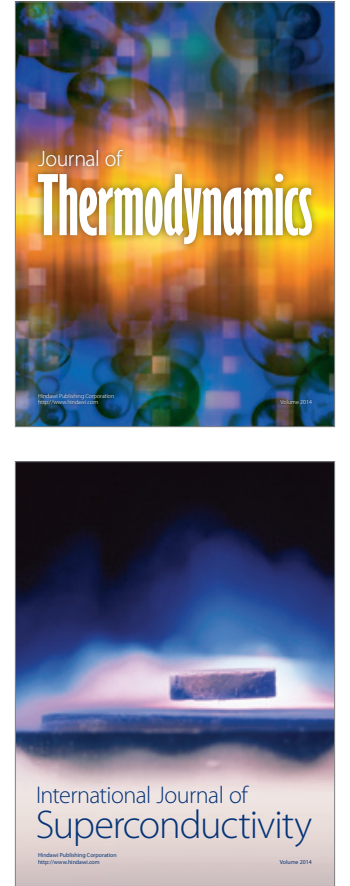
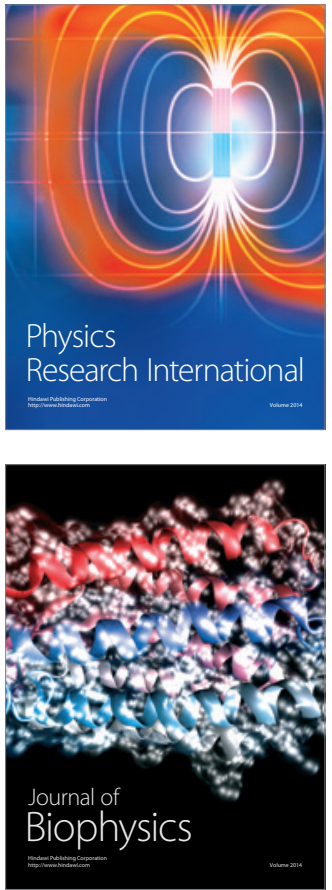
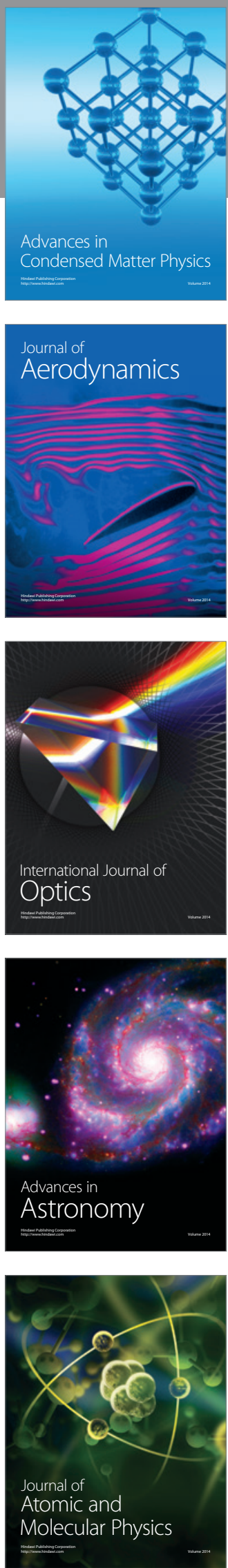